\title{
Effects of finite-range interactions on the one-electron spectral properties of one-dimensional metals: Application to $\mathrm{Bi} / \mathrm{InSb}(001)$
}

\author{
José M. P. Carmelo, ${ }^{1,2,3,4}$ Tilen Čadež ${ }^{5,3}$ Yoshiyuki Ohtsubo, ${ }^{6,7}$ Shin-ichi Kimura,,${ }^{6,7}$ and David K. Campbel1 ${ }^{1}$ \\ ${ }^{1}$ Boston University, Department of Physics, 590 Commonwealth Ave, Boston, Massachusetts 02215, USA \\ ${ }^{2}$ Massachusetts Institute of Technology, Department of Physics, Cambridge, Massachusetts 02139, USA \\ ${ }^{3}$ Center of Physics of University of Minho and University of Porto, P-4169-007 Oporto, Portugal \\ ${ }^{4}$ Department of Physics, University of Minho, Campus Gualtar, P-4710-057 Braga, Portugal \\ ${ }^{5}$ Beijing Computational Science Research Center, Beijing 100193, China \\ ${ }^{6}$ Graduate School of Frontier Biosciences, Osaka University, Suita 565-0871, Japan \\ ${ }^{7}$ Department of Physics, Graduate School of Science, Osaka University, Toyonaka 560-0043, Japan
}

(Received 18 December 2018; revised manuscript received 1 April 2019; published 8 July 2019)

\begin{abstract}
We study the one-electron spectral properties of one-dimensional interacting electron systems in which the interactions have finite range. We employ a mobile quantum impurity scheme that describes the interactions of the fractionalized excitations at energies above the standard Tomonga-Luttinger liquid limit and show that the phase shifts induced by the impurity describe universal properties of the one-particle spectral function. We find the explicit forms in terms of these phase shifts for the momentum dependent exponents that control the behavior of the spectral function near and at the $(k, \omega)$-plane singularities where most of the spectral weight is located. The universality arises because the line shape near the singularities is independent of the short-distance part of the interaction potentials. For the class of potentials considered here, the charge fractionalized particles have screened Coulomb interactions that decay with a power-law exponent $l>5$. We apply the theory to the angle-resolved photo-electron spectroscopy (ARPES) in the highly one-dimensional bismuth-induced anisotropic structure on indium antimonide $\mathrm{Bi} / \mathrm{InSb}(001)$. Our theoretical predictions agree quantitatively with both (i) the experimental value found in $\mathrm{Bi} / \mathrm{InSb}(001)$ for the exponent $\alpha$ that controls the suppression of the density of states at very small excitation energy $\omega$ and (ii) the location in the $(k, \omega)$ plane of the experimentally observed high-energy peaks in the ARPES momentum and energy distributions. We conclude with a discussion of experimental properties beyond the range of our present theoretical framework and further open questions regarding the one-electron spectral properties of $\mathrm{Bi} / \mathrm{InSb}(001)$.
\end{abstract}

DOI: 10.1103/PhysRevB.100.035105

\section{INTRODUCTION}

One-dimensional (1D) interacting systems are characterized by a breakdown of the basic Fermi liquid quasiparticle picture. Indeed, no quasiparticles with the same quantum numbers as the electrons exist when the motion is restricted to a single spatial dimension. Rather, in a 1D lattice, correlated electrons split into basic fractionalized charge-only and spin-only particles $[1,2]$. Hence the removal or addition of electrons generates an energy continuum of excitations described by these exotic fractionalized particles which are not adiabatically connected to free electrons. Hence they must be described using a different language.

These models share common low-energy properties associated with the universal class of the Tomonaga-Luttinger liquid (TLL) [1-4]. To access their high-energy dynamical correlation functions beyond the low-energy TLL limit, approaches such as the pseudofermion dynamical theory (PDT) [5] or the mobile quantum impurity model (MQIM) $[6,7]$ must be used. Those approaches incorporate nonlinearities in the dispersion relations of the fractionalized particles.

An important low-energy TLL property of 1D correlated electronic metallic systems is the universal power-law scaling of the spectral intensity $I(\omega, T)$ such that $I(0, T) \propto T^{\alpha}$ and $I(\omega, 0) \propto|\omega|^{\alpha}$. Here the exponent $\alpha$ controls the suppression of the density of states (SDS) and $\omega$ is a small excitation energy near the ground-state level. The value SDS exponent $\alpha=\left(1-K_{c}\right)^{2} /\left(4 K_{c}\right)$ is determined by that of the TLL charge parameter $K_{c}[1,2,8]$. Importantly, this exponent provides useful information about the range of the underlying electron interactions.

In the case of integrable 1D models solvable by the Bethe ansatz [9] (such as the 1D Hubbard model [10,11]), the PDT and MQIM describe the same mechanisms and lead to the same expressions for the dynamical correlation functions [12]. The advantage of the MQIM is that it also applies to nonintegrable systems [7]. The exponents characterizing the singularities in these systems differ significantly from the predictions of the linear TLL theory, except in the low-energy limit where the latter is valid.

For integrable 1D lattice electronic models with only onsite repulsion (such as the Hubbard model), the TLL charge parameter $K_{c}$ is larger than $1 / 2$ and thus the SDS exponent $\alpha=\left(1-K_{c}\right)^{2} /\left(4 K_{c}\right)$ is smaller than $1 / 8$. In nonintegrable systems, a SDS exponent $\alpha$ larger than $1 / 8$ stems from finiterange interactions [8]. 
TABLE I. Experimental TLL charge parameter $K_{c}=\tilde{\xi}_{c}^{2} / 2$ and related SDS exponent $\alpha=\left(1-K_{c}\right)^{2} /\left(4 K_{c}\right)$ (SI stands for Supplemental Information).

\begin{tabular}{|c|c|c|c|c|}
\hline System & Parameter $K_{c}$ & SDS exponent $\alpha$ & Technique & Source \\
\hline$(\mathrm{TMTSF})_{2} \mathrm{XX}=\mathrm{PF}_{6}, \mathrm{AsF}_{6}, \mathrm{ClO}_{4}$ & 0.23 & 0.64 & Optical conductivity & from SI of Ref. [1] \\
\hline Carbon nanotubes & 0.28 & 0.46 & Photoemission & from SI of Ref. [1] \\
\hline 1D gated semiconductors & $0.26-0.28$ & $0.46-0.53 \approx 0.5$ & Transport conductivity & from SI of Ref. [1] \\
\hline $\mathrm{MoSe}_{2}$ 1D line defects & $0.20-0.22$ & $0.70-0.80$ & ARPES & from Ref. [16] \\
\hline $\mathrm{Bi} / \mathrm{InSb}(001)$ & $0.22-0.24$ & $0.60-0.70$ & ARPES & from Ref. [17] \\
\hline
\end{tabular}

In fact, as shown in Table I, for the metallic states of both 1D and quasi-1D electronic systems, the SDS exponent $\alpha$ frequently has experimental values in the range $0.5-0.8[1,2,8$, 13-18]. In actual materials, a finite effective range interaction [19-23] generally results from screened long-range Coulomb interactions with potentials vanishing as an inverse power of the separation with an exponent larger than one. In general, such finite-range interactions in 1D lattice systems represent a complex and unsolved quantum problem involving nonperturbative microscopic electronic processes. Indeed, as originally formulated, the MQIM does not apply to lattice electronic systems with finite-range interactions whose screened Coulomb potentials vanish as an inverse power of the electron distance.

Recently, the MQIM has been extended to a class of electronic systems with effective interaction ranges of about one lattice spacing, compatible with the high-energy oneelectron spectral properties observed in twin grain boundaries of molybdenum diselenide $\mathrm{MoSe}_{2}$ [16,24]. This has been achieved by suitable renormalization of the phase shifts of the charge fractionalized particles. That theoretical scheme, called here "MQIM-LO," accounts for the effects of only the leading order (LO) in the effective range expansion $[19,20]$ of such phase shifts.

In this paper, we consider a bismuth-induced anisotropic structure on indium antimonide which we henceforth call $\mathrm{Bi} / \mathrm{InSb}(001)$ [17]. Experimentally, strong evidence has been found that $\mathrm{Bi} / \mathrm{InSb}(001)$ exhibits $1 \mathrm{D}$ physics $[17,18]$. However, a detailed understanding of the exotic one-electron spectral properties revealed by its angle resolved photo-emission spectroscopy (ARPES) [17,18] at energy scales beyond the TLL has remained elusive. In particular, the predictions of the MQIM-LO for the location in the $(k, \omega)$ plane of the experimentally observed high-energy peaks in the ARPES momentum distribution curves (MDC) and energy distribution curves (EDC) of $\mathrm{Bi} / \mathrm{InSb}(001)$ do not lead to the same quantitative agreement as for the ARPES in the $\mathrm{MoSe}_{2}$ line defects [16,24]. This raises the important question of what additional effects must be included to obtain agreement with the experimental data.

In this paper, we answer this question by extending the MQIM-LO to a larger class of 1D lattice electronic systems with finite-range interactions by accounting for higher-order terms in the effective range expansion [19,20,25-27] of the phase shifts of the fractionalized charged particles. While the corresponding higher-order "MQIM-HO" corresponds in general to a complicated, nonperturbative many-electron problem, we find, unexpectedly, that the interactions of the fractionalized charged particles with the charge mobile quantum impurity occur in the unitary limit of (minus) infinite scattering length [28-30]. In that limit, the separation between the interacting charged particles (the inverse density) is much greater than the range of the interactions, and the calculations simplify considerably.

The unitary limit plays an important role in the physics of many physical systems, including the dilute neutron matter in shells of neutron stars [31] and in atomic scattering in systems of trapped cold atoms [29,30]. Our discovery of its relevance in a condensed matter system is new and reveals new physics.

The results of the MQIM-HO are consistent with the expectation that the microscopic mechanisms behind the oneelectron spectral properties of $\mathrm{Bi} / \mathrm{InSb}(001)$ include finiterange interactions. Indeed, accounting for the effective range of the corresponding interactions [21-23] leads to theoretical predictions that quantitatively agree with both (i) the experimental value of the SDS exponent $(\alpha \in[0.6-0.7])$ in $\mathrm{Bi} / \mathrm{InSb}(001)$ observed in $I(\omega, 0) \propto|\omega|^{\alpha}$ and (ii) the location in the $(k, \omega)$ plane of the experimentally observed high-energy peaks in the ARPES MDC and EDC.

Since $\mathrm{Bi} / \mathrm{InSb}(001)$ is a complex system and the MQIMHO predictions are limited to the properties (i) and (ii), in the discussion section of this paper we consider other possible effects beyond the present theoretical framework that might contribute to the microscopic mechanisms determining spectral properties of $\mathrm{Bi} / \mathrm{InSb}(001)$.

In this paper, we employ units of $\hbar=1$ and $k_{B}=1$. In Sec. II, we introduce the theoretical scheme used in our studies. The effective-range expansion of the phase shift associated with the interactions of the charge fractionalized particles and charge hole mobile impurity, the corresponding unitary limit, and the scattering lengths are all issues we address in Sec. III. In Sec. IV, the effective range expression is derived and expressed in terms of the ratio of the renormalized and bare scattering lengths. In Sec. V, we show how our approach predicts the location in the $(k, \omega)$ plane of the experimentally observed high-energy $\mathrm{Bi} / \mathrm{InSb}(001)$ ARPES MDC and EDC peaks. In Sec. VI, we discuss our results and experimental properties outside the present theoretical framework, mention open questions on the $\mathrm{Bi} / \mathrm{InSb}(001)$ spectral properties, and offer concluding remarks. 


\section{THE MODEL}

The 1D model Hamiltonian associated with the MQIM-HO for electronic density $\left.n_{e} \in\right] 0,1[$ is given by

$$
\begin{aligned}
& \hat{H}=t \hat{T}+\hat{V}, \quad \text { where } \\
& \hat{T}=-\sum_{\sigma=\uparrow, \downarrow} \sum_{j=1}^{L}\left(c_{j, \sigma}^{\dagger} c_{j+1, \sigma}+c_{j+1, \sigma}^{\dagger} c_{j, \sigma}\right), \\
& \hat{V}=\sum_{r=0}^{L / 2-1} V_{e}(r) \sum_{\sigma=\uparrow, \downarrow} \sum_{\sigma^{\prime}=\uparrow, \downarrow} \sum_{j=1}^{L} \hat{\rho}_{j, \sigma} \hat{\rho}_{j+r, \sigma^{\prime}} .
\end{aligned}
$$

Here, $\hat{\rho}_{j, \sigma}=\left(c_{j, \sigma}^{\dagger} c_{j, \sigma}-\frac{1}{2}\right), V_{e}(0)=U / 2, V_{e}(r)=U F_{e}(r) / r$ for $r>0$, and $F_{e}(r)$ is a continuous screening function such that $F_{e}(r) \leqslant 1 / 4$, which at large $r$ vanishes as some inverse power of $r$ whose exponent is larger than one, so that $\lim _{r \rightarrow \infty} F_{e}(r)=0$.

We use a representation of the fractionalized $c$ (charge) and $s$ (spin) particles that also naturally emerges in the MQIM-LO [16]. For simplicity, in general in this paper they are called $c$ particles and $s$ particles, respectively. They occupy a $c$ band and an $s$ band whose momentum values $q_{j}$ and $q_{j}^{\prime}$, respectively, are such that $q_{j+1}-q_{j}=2 \pi / L$ and $q_{j+1}^{\prime}-q_{j}^{\prime}=2 \pi / L$. In the thermodynamic limit, one often uses a continuum representation in terms of corresponding $c$ band momentum variables $q$ and $s$ band momentum variables $q^{\prime}$ with ground-state occupancies $q \in\left[-2 k_{F}, 2 k_{F}\right]$ and $q^{\prime} \in\left[-k_{F}, k_{F}\right]$, respectively, where $2 k_{F}=\pi n_{e}$. The energy dispersions for $c$ and $s$ particles, $\tilde{\varepsilon}_{c}(q)$ and $\tilde{\varepsilon}_{s}\left(q^{\prime}\right)$, respectively, are defined for these momentum intervals in Eqs. (A2) and (A4)-(A10) of Appendix A.

Most of the weight of the one-electron spectral function is generated by transitions to excited states involving creation of one hole in the $c$ band, one hole in the $s$ band, plus low-energy particle-hole processes in such bands. Processes where both holes are created away from the $c$ band and $s$ band Fermi points $\pm 2 k_{F}$ and $\pm k_{F}$, respectively, contribute to the spectral-function continuum. Processes where the $c$ band hole is created at momentum values spanning its band interval $q \in]-2 k_{F}, 2 k_{F}$ [ and the $s$ hole (spinon) is created near one of its Fermi points $\pm k_{F}$ contribute to the $c$ and $c^{\prime}$ branch lines whose spectra run from $k \in]-k_{F}, k_{F}[$ and $k \in]-3 k_{F}, 3 k_{F}$, respectively. Since in such processes the $c$ band hole is created away from the $c$ band Fermi points, we call it a $c$ (charge) hole mobile impurity. Finally, processes where the $s$ band hole is created at momentum values in the interval $\left.q^{\prime} \in\right]-k_{F}, k_{F}$ [ and the $c$ hole (holon) is created near one of its Fermi points $\pm 2 k_{F}$ contribute to the $s$ branch line whose spectrum runs from $k \in]-k_{F}, k_{F}$ [. In the case of these processes it is the $s$ band hole that is created away from the corresponding $s$ band Fermi points. Hence we call it $s$ (spin) hole mobile impurity. See a sketch of such spectra in Fig. 1. In the remainder of this paper, the charge (and spin) hole mobile impurity is merely called $c$ (and $s$ ) impurity.

The one-electron operators matrix elements between energy eigenstates in the expressions for the spectral function involve phase shifts and the charge parameter $\tilde{\xi}_{c}=\sqrt{2 K_{c}}$ whose value is determined by them. Its range for the present lattice systems is $\left.\tilde{\xi}_{c}=\sqrt{2 K_{c}} \in\right] 1 / 2, \xi_{c}$ ], where the bare parameter

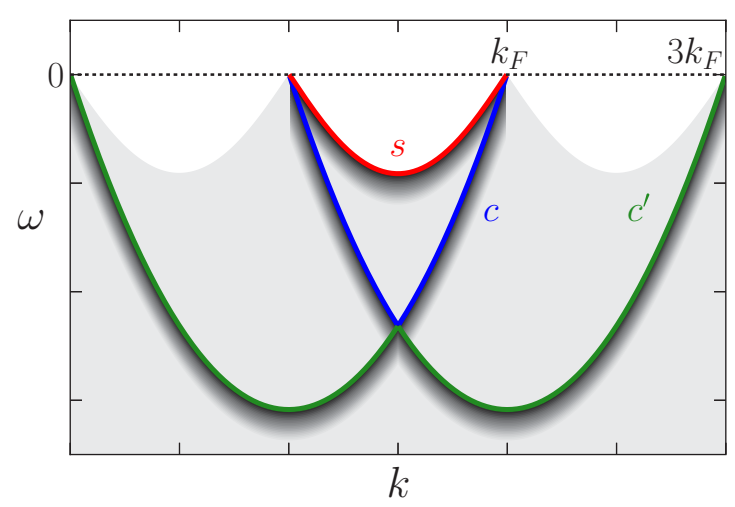

FIG. 1. Sketch of the $s$ (spin) and $c$ and $c^{\prime}$ (charge) branch lines in the one-electron removal spectral function of the lattice electronic correlated models discussed in this paper. The soft grey region refers to the small spectral-weight distribution continuum whereas the darker grey regions below the branch lines typically display more weight. In the actual spectral function, Eq. (2), this applies to $k$ subdomains for which the exponents that control the line shape near those lines are negative. The lack of spectral weight in some of the figure $(k, \omega)$-plane regions is imposed by kinematical constraints.

$\left.\xi_{c} \in\right] 1, \sqrt{2}[$ defined by Eq. (A16) of Appendix A refers to the 1D Hubbard model. Note that the model in Eq. (1), becomes the 1D Hubbard model at the bare charge parameter value, $\tilde{\xi}_{c}=\xi_{c}$. In this limit, the SDS exponent reads $\alpha_{0}=(2-$ $\left.\xi_{c}^{2}\right)^{2} /\left(8 \xi_{c}^{2}\right) \in[0,1 / 8]$ with $\alpha_{0}=0$ and $\alpha_{0}=1 / 8$ for $u \rightarrow 0$ and $u \rightarrow \infty$, respectively. For $\left.n_{e} \in\right] 0,1\left[\right.$, there is a $\xi_{c} \rightarrow \tilde{\xi}_{c}$ transformation [16] for each fixed value of $\xi_{c}$ and $\tilde{\xi}_{c}<\xi_{c}$ such that $\left.\xi_{c} \in\right] 1, \sqrt{2}\left[\right.$ and $\left.\left.\tilde{\xi}_{c} \in\right] 1 / 2,1[;] 1, \xi_{c}\right]$. This maps the 1D Hubbard model onto the model, Eq. (1), upon gently turning on $F_{e}(r)$. Consistent with this result, $\lim _{\tilde{\xi}_{c} \rightarrow \xi_{c}} F_{e}(r) \rightarrow 0$ for $r \in[0, \infty]$. For $\tilde{\xi}_{c}<\xi_{c}$, the corresponding SDS exponent intervals are $\alpha=\left(2-\tilde{\xi}_{c}^{2}\right)^{2} /\left(8 \tilde{\xi}_{c}^{2}\right) \in\left[\alpha_{0}, 1 / 8[;] 1 / 8,49 / 32[\right.$.

The phase shifts in the one-electron matrix elements play a major role in our study by appearing explicitly in the expressions of the momentum-dependent exponents of the one-electron removal spectral function. These phases shifts are $2 \pi \tilde{\Phi}_{c, s}\left( \pm 2 k_{F}, q^{\prime}\right)$ and $2 \pi \tilde{\Phi}_{c, c}\left( \pm 2 k_{F}, q\right)$. Specifically, $-2 \pi \tilde{\Phi}_{c, s}\left( \pm 2 k_{F}, q^{\prime}\right)$ and $-2 \pi \tilde{\Phi}_{c, c}\left( \pm 2 k_{F}, q\right)$ are the phase shifts, respectively, imposed on a $c$ particle of $c$ band momentum $\pm 2 k_{F}$ by a $s$ and $c$ impurity created at momentum $q^{\prime} \in\left[-k_{F}, k_{F}\right]$ and $q \in\left[-2 k_{F}, 2 k_{F}\right]$. (Their explicit expressions are given below). The charge parameter $\tilde{\xi}_{c}$ is given by a superposition of charge-charge phase shifts,

$$
\tilde{\xi}_{c}=1+\lim _{q \rightarrow 2 k_{F}}\left\{\tilde{\Phi}_{c, c}\left(+2 k_{F}, q\right)+\tilde{\Phi}_{c, c}\left(-2 k_{F}, q\right)\right\} .
$$

The expressions for the exponents of spectral functions also involve the phase shifts $2 \pi \tilde{\Phi}_{s, c}\left( \pm k_{F}, q\right)=\mp \frac{\pi}{\sqrt{2}}$ and $2 \pi \tilde{\Phi}_{s, s}\left( \pm k_{F}, q^{\prime}\right)= \pm(\sqrt{2}-1)\left(\sqrt{2}+(-1)^{\delta_{q^{\prime} \pm k_{F}}}\right) \frac{\pi}{\sqrt{2}}$ induced on a $s$ particle of $s$ band momentum $\pm k_{F}$ by a $c$ and $s$ impurity created at momentum $q \in\left[-2 k_{F}, 2 k_{F}\right]$ and $q^{\prime} \in\left[-k_{F}, k_{F}\right]$, respectively. Their simple expressions are invariant under the $\xi_{c} \rightarrow \tilde{\xi}_{c}$ transformation and, due to the spin SU(2) symmetry, are interaction, density, and momentum independent. (Except for $(-1)^{\delta_{q^{\prime}, \pm k_{F}}}$ in the $2 \pi \tilde{\Phi}_{s, s}\left( \pm k_{F}, q^{\prime}\right)$ expression at $\left.q^{\prime}= \pm k_{F}\right)$.

For small energy deviations $\left(\tilde{\omega}_{\beta}(k)-\omega\right)>0$ and $\left(\tilde{\omega}_{s}(k)-\right.$ $\omega)>0$ near the $\beta=c, c^{\prime}$ branch lines and $s$ branch line, the 
spectral function behaves as

$$
\begin{aligned}
& \tilde{B}(k, \omega) \approx \sum_{\iota= \pm 1} C_{\beta, l} \operatorname{Im}\left\{\left(\frac{(\iota)}{\tilde{\omega}_{\beta}(k)-\omega-\frac{i}{2 \tau_{\beta}(k)}}\right)^{-\tilde{\zeta}_{\beta}(k)}\right\}, \\
& \tilde{B}(k, \omega)=C_{s}\left(\tilde{\omega}_{s}(k)-\omega\right)^{\tilde{\zeta}_{s}(k)},
\end{aligned}
$$

respectively. Here $C_{\beta, \iota}$ and $C_{s}$ are $n_{e}, u=U / 4 t$, and $\tilde{\xi}_{c}$ dependent constants for energy and momentum values corresponding to the small energy deviations $\left(\tilde{\omega}_{\beta}(k)-\omega\right)>0$ and $\left(\tilde{\omega}_{s}(k)-\omega\right)>0$, respectively, and $\omega<0$ are high energies beyond those of the TLL.

The upper bounds of the constants $C_{c, l}, C_{c^{\prime}, l}$, and $C_{s}$ in Eq. (2) are known from matrix elements and sum rules for spectral weights, but their precise values remain in general an unsolved problem. The expressions for the $\gamma=c, c^{\prime}, s$ spectra $\tilde{\omega}_{\gamma}(k)$ and exponents $\tilde{\zeta}_{\gamma}(k)$ are given in Eqs. (A1) and (A3) of Appendix A, respectively. As discussed in Appendix B, the MQIM-HO also applies to the low-energy TLL limit in which such exponents have different expressions. For the present high-energy regime, they have the same expressions as for the MQIM-LO except that the phase shift $2 \pi \tilde{\Phi}_{c, c}\left( \pm 2 k_{F}, q\right)$ in that of the spectral function exponents $\tilde{\zeta}_{c}(k)$ and $\tilde{\zeta}_{c^{\prime}}(k)$ has MQIM-HO additional terms.

That the $s$ branch line coincides with the edge of the support for the spectral function ensures that near it the line shape is power-law-like, as given in Eq. (2). For the $c, c^{\prime}$ branch likes, which run within the spectral weight continuum, the $\beta=c, c^{\prime}$ lifetime $\tau_{\beta}(k)$ in Eq. (2) is very large for the interval $\left.\tilde{\xi}_{c} \in\right] 1, \xi_{c}$, so that the expression given in that equation is nearly power-law-like, $\tilde{B}(k, \omega) \propto\left(\tilde{\omega}_{\beta}(k)-\omega\right)^{\tilde{\zeta}_{\beta}(k)}$. The finite-range interaction effects increase upon decreasing $\tilde{\xi}_{c}$ in the interval $\tilde{\xi}_{c} \in\left[\tilde{\xi}_{c}^{\ominus}, 1\left[\right.\right.$ where $\tilde{\xi}_{c}^{\ominus}=1 / \xi_{c}$. In it the corresponding $c$ impurity relaxation processes associated with large lifetimes $\tau_{c}(k)$ and $\tau_{c^{\prime}}(k)$ in Eq. (2) for the $k$ intervals for which $\tilde{\zeta}_{c}(k)<0$ and $\tilde{\zeta}_{c^{\prime}}(k)<0$, respectively, start transforming the power-law singularities into broadened peaks with small widths. Such effects become more pronounced upon further decreasing $\tilde{\xi}_{c}$ in the interval $\left.\left.\tilde{\xi}_{c} \in\right] \tilde{\xi}_{c}^{\ominus}, 1\right]$. As discussed in more detail below in Sec. V D, for $k$ ranges for which the exponents $\tilde{\zeta}_{c}(k)$ and $\tilde{\zeta}_{c^{\prime}}(k)$ become positive upon decreasing $\tilde{\xi}_{c}$, the relaxation processes wash out the peaks entirely.

\section{THE EFFECTIVE-RANGE EXPANSION AND THE UNITARY LIMIT}

\section{A. The effective-range expansion}

As we shall establish in detail below, the finite-range electron interactions have their strongest effects in the chargecharge interaction channel. In contrast, for the charge-spin channel, the renormalization factor of the phase shift,

$$
2 \pi \tilde{\Phi}_{c, s}\left( \pm 2 k_{F}, q^{\prime}\right)=\frac{\tilde{\xi}_{c}}{\xi_{c}} 2 \pi \Phi_{c, s}\left( \pm 2 k_{F}, q^{\prime}\right),
$$

remains that of the MQIM-LO.

For small relative momentum $k_{r}=q \mp 2 k_{F}$ of the $c$ impurity of momentum $q$ and $c$ particle of momentum $\pm 2 k_{F}$ the phase shift $\tilde{\Phi}_{c}\left(k_{r}\right)=-2 \pi \tilde{\Phi}_{c, c}\left( \pm 2 k_{F}, \pm 2 k_{F}+k_{r}\right)$ associated with the charge-charge channel obeys an effective range expansion,

$\cot \left(\tilde{\Phi}_{c}\left(k_{r}\right)\right)=\frac{-1}{\tilde{a} k_{r}}+\frac{1}{2} R_{\text {eff }} k_{r}-P_{\text {eff }} R_{\text {eff }}^{3} k_{r}^{3}+\mathcal{O}\left(k_{r}^{5}\right)$.

This equation is the same as for three-dimensional (3D) swave scattering problems if $k_{r}$ is replaced by $\left|k_{r}\right|[19,20]$. The first and second terms involve the scattering length $\tilde{a}$ and effective range $R_{\text {eff }}$, respectively. The third and higher terms are negligible and involve the shape parameters $[19,20$, 25-27].

One finds that in the bare charge parameter limit, $\tilde{\xi}_{c}=\xi_{c}$, the effective range expansion reads $\cot \left(\Phi_{c}\left(k_{r}\right)\right)=$ $-1 /\left(a k_{r}\right) \quad$ where $\quad \Phi_{c}\left(k_{r}\right)=-2 \pi \Phi_{c, c}\left( \pm 2 k_{F}, \pm 2 k_{F}+k_{r}\right)$, $2 \pi \Phi_{c, c}\left( \pm 2 k_{F}, q\right)$ is the bare phase shift defined in Eqs. (A11)-(A15) of Appendix A, and $a=\lim _{\tilde{\xi}_{c} \rightarrow \xi_{c}} \tilde{a}$ is the bare scattering length.

Due to the 1D charge-spin separation at all MQIM energy scales, the repulsive electronic potential $V_{e}(r)$ gives rise to an attractive potential $V_{c}(x)$ associated with the interaction of the $c$ particle and $c$ impurity at a distance $x$. To go beyond the MQIM-LO, we must explicitly account for the general properties of $V_{c}(x)$ whose form is determined by that of $V_{e}(r)$. The corresponding relation between the electron and $c$ particle representations is discussed see Appendix C. The attractive potential $V_{c}(x)$ is negative for $x>x_{0}$ where $x_{0}$ is a nonuniversal distance that either vanishes or is much smaller than the lattice spacing $a_{0}$. Moreover, for the present class of systems $V_{c}(x)$ vanishes for large $x$ as

$$
\begin{aligned}
V_{c}^{\text {asy }}(x) & =-\frac{\gamma_{c}}{x^{l}}=-\frac{C_{c}}{\left(x / 2 r_{l}\right)^{l}}, \quad \text { where } \\
C_{c} & =\frac{1}{\left(2 r_{l}\right)^{2} \mu} \quad \text { and } \quad \gamma_{c}=\frac{\left(2 r_{l}\right)^{l-2}}{\mu} .
\end{aligned}
$$

Here, $\mu$ is a nonuniversal reduced mass, $l$ is an integer determined by the large- $r$ behavior of $V_{e}(r)$, and $2 r_{l}$ is a length scale whose $l$ dependence for $\tilde{\xi}_{c}<1$ is given below in Sec. IV. (And is twice the van der Waals length at $l=6$ ).

Since $V_{c}(x)$ has asymptotic behavior $1 / x^{l}$, the scattering length, effective range, and shape parameter terms in Eq. (4) only converge if $l>5, l>7$, and $l>9$, respectively [27]. We shall find that agreement with the experimental results is achieved provided that the effective range studied in Sec. IV is finite and this requires that $l>5$ in Eq. (5).

Similarly to the potentials considered in Refs. [23,32], the class of potentials with large-distance behavior, Eq. (5) and whose depth is larger that the scattering energy of the corresponding interactions considered here are such that the positive "momentum" $\sqrt{2 \mu\left(-V_{c}(x)\right)}$ obeys a sum rule of general form:

$$
\begin{aligned}
\int_{x_{0}}^{\infty} d x \sqrt{2 \mu\left(-V_{c}(x)\right)} & =\Phi+\frac{\theta_{c} \pi}{2(l-2)}, \quad \text { where } \\
\tan (\Phi) & =-\frac{\Delta a}{\tilde{a}} \cot \left(\frac{\pi}{l-2}\right) \text { and thus } \\
\frac{a}{\tilde{a}} & =1-\tan \left(\frac{\pi}{l-2}\right) \tan (\Phi)
\end{aligned}
$$

Here, $\Delta a / \tilde{a}$ where $\Delta a=a-\tilde{a}$ is a relative fluctuation that involves two uniquely defined yet nonuniversal scattering 
lengths, $a$ and $\tilde{a}$. As justified in Sec. IV, in the present unitarylimit case discussed in Sec. III B, they are the bare and renormalized scattering lengths, respectively, defined in that section. The physically important renormalized charge parameter range is $\left.\tilde{\xi}_{c} \in\right] 1 / 2,1$ [ for which $\alpha>1 / 8$. The term $\theta_{c} \pi /[2(l-$ $2)]$ in Eq. (6) refers to a potential boundary condition $[23,32]$ with $\theta_{c}=1$ for that interval. (In that regime, the expressions in Eq. (6) are similar to those in Eqs. (4) and (6) of Ref. [23] with $a, \tilde{a}, l$, and $\Phi$ corresponding to $a, \bar{a}, n$, and $\Phi-\pi /[2(n-2)]$, respectively). A function $\theta_{c}=\sqrt{\left(\xi_{c}^{4}-\tilde{\xi}_{c}^{4}\right) /\left(\xi_{c}^{4}-1\right)}$ for the interval $\tilde{\xi}_{c} \in$ ] $1, \xi_{c}$ [ for which $\alpha<1 / 8$ merely ensures that the sum rule in Eq. (6) continuously vanishes as $\tilde{\xi}_{c} \rightarrow \xi_{c}$.

Our choice of potentials with large- $x$ behavior given in Eq. (5) is such that the sum rule, Eq. (6), is obeyed yet for small $x$ the form of $V_{c}(x)$ is not universal and is determined by the specific small- $r$ form of $V_{e}(r)$ itself. The zero-energy phase $\Phi$ in Eq. (6) whose physics is further clarified below can be expressed as

$$
\Phi=\int_{x_{0}}^{x_{2}} d x \sqrt{2 \mu\left(-V_{c}(x)\right)} \quad \text { where } \quad x_{2}=2 r_{l}\left(\frac{4 \sqrt{2}}{\pi \theta_{c}}\right)^{\frac{2}{l-2}}
$$

Indeed, $\quad V_{c}(x)=V_{c}^{\text {asy }}(x)$ for $\quad x>x_{2}$ and $\int_{x_{2}}^{\infty} d x$ $\sqrt{2 \mu\left(-V_{c}^{\text {asy }}(x)\right)}=\frac{\theta_{c} \pi}{2(l-2)}$. Here, $x_{2} \approx 2 r_{l}$ for $\left.\tilde{\xi}_{c} \in\right] 1 / 2,1[$ with the ratio $x_{2} / 2 r_{l}$ decreasing from 1.342 at $l=6$ to 1 at $l=\infty$. For $\left.\left.\tilde{\xi}_{c} \in\right] 1, \xi_{c}\right]$, it is an increasing function of $\tilde{\xi}_{c}$ such that $\lim _{\tilde{\xi}_{c} \rightarrow \xi_{c}} x_{2} / 2 r_{l}=\infty$ for $l$ finite.

The universal form of the spectral function near the singularities, Eq. (2), is determined by the large $x$ behavior of $V_{c}(x)$, Eq. (5), and sum rules, Eqs. (6) and (7). In the spectral-weight continuum, its form is not universal, as it depends on the specific small $x$ form of $V_{c}(x)$ determined by $V_{e}(r)$.

The length scale $2 r_{l}$ in Eq. (5) is found below in Sec. IV to obey the inequality $\sqrt{2}\left(2 r_{l}\right)^{\frac{l-2}{2}}=\sqrt{2 \mu \gamma_{c}} \gg 1$ in units of $a_{0}=1$. $\left(\sqrt{2 \mu \gamma_{c}}\right.$ in such units corresponds to the important parameter $\gamma=\sqrt{2 \mu \alpha} / \hbar$ of Ref. [23] in units of Bohr radius $a_{0}=0.529177$ Itextrm $\AA$ with $\mu$ and $\alpha$ corresponding to $\mu$ and $\gamma_{c}$, respectively). This inequality justifies why $V_{c}(x)=$ $V_{c}^{\text {asy }}(x)$ for $x>x_{2}$ and implies that $\int_{x_{0}}^{x_{2}} d x \sqrt{2 \mu\left(-V_{c}(x)\right)}$ in Eq. (7) has for $\left.\tilde{\xi}_{c} \in\right] 1 / 2,1$ [ large values. This is consistent with the above mentioned requirement of the scattering energy of the residual interactions of the $c$ particles and $c$ impurity being smaller than the depth $-V_{c}\left(x_{1}\right)$ of the potential $V_{c}(x)$ well, which since $\int_{x_{0}}^{\infty} d x \sqrt{2 \mu\left(-V_{c}(x)\right)} / \pi \gg 1$ must be large. Here, $x_{1}$ is a small nonuniversal potential-dependent $x$ value such that $x_{0}<x_{1}<a_{0}$ at which $\partial V_{c}(x) / \partial x=0$ and $-V_{c}(x)$ reaches its maximum value.

\section{B. The unitary limit and the scattering lengths}

As confirmed below in Sec. IV, the expression for the phase shift in the thermodynamic limit,

$$
-\left.2 \pi \tilde{\Phi}_{c, c}\left( \pm 2 k_{F}, \pm 2 k_{F}+k_{r}\right)\right|_{k_{r}=\mp \frac{2 \pi}{L}}=\mp \frac{\left(\tilde{\xi}_{c}-1\right)^{2} \pi}{\tilde{\xi}_{c}},
$$

for $\lim _{k_{r} \rightarrow 0} \tilde{\Phi}_{c}\left(k_{r}\right)$ remains the same as for the MQIM-LO. Its use along with that of $\mp\left(\xi_{c}-1\right)^{2} \pi / \xi_{c}$ for the bare phase shift $\lim _{k_{r} \rightarrow 0} \cot \left(\Phi_{c}\left(k_{r}\right)\right)$ in the leading term of the corresponding effective-range expansions gives the scattering lengths. In the thermodynamic limit, they read

$$
\begin{aligned}
& \tilde{a}=-\frac{L}{2 \pi} \tan \left(\frac{\left(\tilde{\xi}_{c}-1\right)^{2} \pi}{\tilde{\xi}_{c}}\right) \rightarrow-\infty \text { for } \quad \tilde{\xi}_{c} \neq 1 \text { and } \\
& a=-\frac{L}{2 \pi} \tan \left(\frac{\left(\xi_{c}-1\right)^{2} \pi}{\xi_{c}}\right) \rightarrow-\infty \text { for } \xi_{c} \neq 1,
\end{aligned}
$$

respectively. This is known as the unitary limit [29,30].

The validity of the MQIM-HO refers to this limit, which occurs provided that $\xi \neq 1, \tilde{\xi}_{c} \neq 1$, and as confirmed below that $\tilde{\xi}_{c}>1 / 2$. The dependence of the bare charge parameter $\xi_{c}=\xi_{c}\left(n_{e}, u\right)$ on the density $n_{e}$ and $u=U / 4 t$ is defined by Eq. (A16) of Appendix A. It is such that $\xi_{c}=\sqrt{2}$ for $u \rightarrow 0$ and $\xi_{c}=1$ for $u \rightarrow \infty$ for $\left.n_{e} \in\right] 0,1\left[\right.$ and $\xi_{c}=1$ for $u>0$ and $\xi_{c}=\sqrt{2}$ at $u=0$ for both $n_{e} \rightarrow 0$ and $n_{e} \rightarrow 1$. This implies that $a=-\infty$ provided that the relative momentum obeys the inequality $\left|k_{r}\right| \ll \frac{\tan \left(\pi n_{e}\right)}{4 u}$. This excludes electronic densities very near $n_{e}=0$ and $n_{e}=1$ for all $u$ values and excludes large $u$ values for the remaining electronic densities.

The phase shifts $\tilde{\Phi}_{c}=-2 \pi \tilde{\Phi}_{c, c}\left( \pm 2 k_{F}, q\right)$ incurred by the $c$ particles from their interactions with the $c$ impurity created at momentum $q \in\left[-2 k_{F}+k_{F c}^{0}, 2 k_{F}-k_{F c}^{0}\right.$ [ have $c$ band momenta in two small intervals $\left[-2 k_{F},-2 k_{F}+k_{F c}^{0}\right]$ and $\left[2 k_{F}-k_{F c}^{0}, 2 k_{F}\right]$ near the $c$ band Fermi points $-2 k_{F}$ and $2 k_{F}$, respectively. As discussed in Appendix B, the creation of an impurity in the $c$ band intervals $q \in\left[-2 k_{F},-2 k_{F}+k_{F c}^{0}\right]$ and $q \in\left[2 k_{F}-k_{F c}^{0}, 2 k_{F}\right]$ refers to the low-energy TLL regime. Its velocity becomes that of the low-energy particle-hole excitations near $-2 k_{F}$ and $2 k_{F}$, respectively. In this regime, the physics is different, as the impurity loses its identity, since it cannot be distinguished from the $c$ band holes (TLL holons) in such excitations.

The small momentum $k_{F c}^{0}$ can be written as $k_{F c}^{0}=$ $\pi n_{F c}^{0}$. The unitary limit refers to the corresponding low density $n_{F c}^{0}$ of $c$ particle scatterers with phase shift $\tilde{\Phi}_{c}=-2 \pi \tilde{\Phi}_{c, c}\left( \pm 2 k_{F}, q\right)$ and $c$ band momentum values $\left[-2 k_{F},-2 k_{F}+k_{F c}^{0}\right]$ and $\left[2 k_{F}-k_{F c}^{0}, 2 k_{F}\right]$ near $-2 k_{F}$ and $2 k_{F}$, respectively. They, plus the single $c$ impurity constitute the usual dilute quantum liquid of the unitary limit whose density is thus $n_{F c}^{0}$. $k_{F c}^{0}$ is such that $k_{F c}^{0}|\tilde{a}|=\frac{1}{2} N_{F c}^{0} \tan \left(\left[\left(\tilde{\xi}_{c}-\right.\right.\right.$ $\left.\left.1)^{2} / \tilde{\xi}_{c}\right] \pi\right)$ and $k_{F c}^{0}|a|=\frac{1}{2} N_{F c}^{0} \tan \left(\left[\left(\xi_{c}-1\right)^{2} / \xi_{c}\right] \pi\right)$ for $\tilde{\xi}_{c}=$ $\xi_{c}$. Here, $N_{F c}^{0}$ is the number of $c$ particle scatterers in $n_{F c}^{0}=$ $N_{F c}^{0} / L$.

In the thermodynamic limit, one has that $n_{F_{c}}^{0}$ is very small or even such that $\lim _{L \rightarrow \infty} n_{F c}^{0} \rightarrow 0$. Consistent with this result, the following relations of the usual unitary limit of the dilute quantum liquid unitary limit [29], $R_{\text {eff }} \ll 1 / k_{F c}^{0} \ll|\tilde{a}|$ and $0 \ll 1 / k_{F c}^{0} \ll|a|$ hold. The effective range $R_{\text {eff }}$ derived in Sec. IV is such that $R_{\text {eff }} \rightarrow \infty$ as $\tilde{\xi}_{c} \rightarrow 1 / 2$. The unitary limit requirement that $R_{\text {eff }} \ll 1 / k_{F c}^{0}$ in the thermodynamic limit is the reason that the value $\tilde{\xi}_{c}=1 / 2$ is excluded from the regime in which the MQIM-HO is valid.

Importantly, although both $a^{-1}=0$ and $\tilde{a}^{-1}=0$, the ratio $\tilde{a} / a$ is finite. Since below in Sec. IV we confirm that $a$ and $\tilde{a}^{-1}$ are in Eq. (6) the scattering lengths given by Eq. (9), the value of $\tilde{\xi}$ in their ratio $\tilde{a} / a$ expression is found to be controlled by the potential $V_{c}(x)$ though $\tan (\Phi)$ in the sum rules provided in 


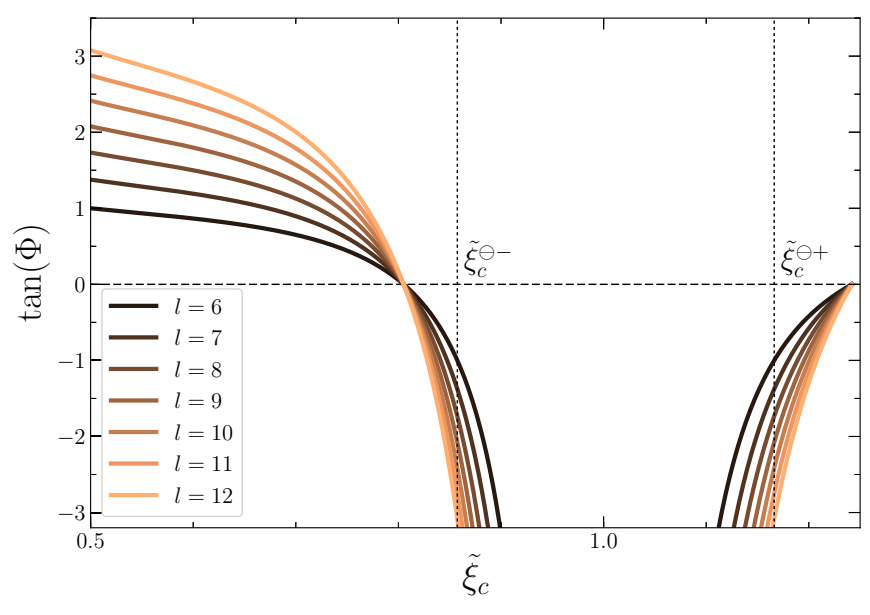

FIG. 2. $\tan (\Phi)=-(\Delta a / \tilde{a}) \cot (\pi /[l-2])$, Eqs. (6) and (11), as a function of the renormalized charge parameter $\tilde{\xi}_{c}$ for the electronic density $n_{e}=0.176$, interaction $u=U / 4 t=0.30$, and integer quantum numbers $l=6-12$ used in $\mathrm{Sec}$. $\mathrm{V}$ for $\mathrm{Bi} / \mathrm{InSb}(001)$. For $\tilde{\xi}_{c} \rightarrow 1 / 2$ and at $\tilde{\xi}_{c}=\tilde{\xi}_{c}^{\ominus}=1 / \xi_{c}=0.805, \tan (\Phi)$ reads $\cot (\pi /(l-$ $2))$ and 0 , respectively, and at both $\tilde{\xi}_{c}=\tilde{\xi}_{c}^{\ominus-}=0.857$ and $\tilde{\xi}_{c}=$ $\tilde{\xi}_{c}^{\ominus+}=1.166$ it is given by $-\cot (\pi /(l-2))$. The MQIM-HO is not valid near $\tilde{\xi}_{c}=1$ at which $\Delta a / \tilde{a}=\infty$ and the corresponding scattering problem does not refer to the unitary limit. The finite-range effects are more pronounced for $\left.\tilde{\xi}_{c} \in\right] 1 / 2, \tilde{\xi}_{c}^{\ominus}[$ when $\Delta a / \tilde{a}<0$ and $\tan (\Phi)>0$.

Eqs. (6) and (7) as

$$
\frac{\tilde{a}}{a}=\frac{\tan \left(\pi\left(\tilde{\xi}_{c}-1\right)^{2} / \tilde{\xi}_{c}\right)}{\tan \left(\pi\left(\xi_{c}-1\right)^{2} / \xi_{c}\right)}=\frac{1}{1-\tan \left(\frac{\pi}{l-2}\right) \tan (\Phi)} .
$$

The first expression on the right-hand side of this equation is specific to the present $1 \mathrm{D}$ quantum problem and follows directly from Eq. (9). Hence, in the present case, $\tan (\Phi)$ in Eq. (6) can be expressed as

$$
\tan (\Phi)=-\frac{\sin \left(\frac{\left(\xi_{c}-\tilde{\xi}_{c}\right)\left(\xi_{c} \tilde{\xi}_{c}-1\right) \pi}{\xi_{c} \tilde{\xi}_{c}}\right) \cot \left(\frac{\pi}{l-2}\right)}{\sin \left(\frac{\left(\tilde{\xi}_{c}-1\right)^{2}}{\tilde{\xi}_{c}} \pi\right) \cos \left(\frac{\left(\xi_{c}-1\right)^{2}}{\xi_{c}} \pi\right)}
$$

One finds from the use of Eq. (10) that effects of the finite-range interactions controlled by relative fluctuation $\Delta a / \tilde{a}$ in $\tan (\Phi)=-\frac{\Delta a}{\tilde{a}} \cot \left(\frac{\pi}{l-2}\right)$, Eq. (6), are stronger for $\left.\left.\left.\left.\tilde{\xi}_{c} \in\right] 1 / 2, \tilde{\xi}_{c}^{\oslash}\right]=\right] 1 / 2,1 / \xi_{c}\right]$ when $\Delta a / \tilde{a}<0, \tilde{a} / a>1$, and $\tan (\Phi)>0$ in Fig. 2. Upon increasing $\tilde{\xi}_{c}$ within the intervals $\left.\tilde{\xi}_{c} \in\right] 1 / 2,1\left[\right.$ and $\left.\tilde{\xi}_{c} \in\right] 1, \xi_{c}$ ], the relative fluctuation increases from $\Delta a / \tilde{a}=-1$ for $\tilde{\xi}_{c} \rightarrow 1 / 2$ to $\Delta a / \tilde{a}=\infty$ for $\tilde{\xi}_{c} \rightarrow 1$, crossing 0 and 1 at $\tilde{\xi}_{c}=\tilde{\xi}_{c}^{\ominus}=1 / \xi_{c}$ and $\tilde{\xi}_{c}=\tilde{\xi}_{c}^{\ominus-}$, respectively. Upon further increasing $\tilde{\xi}_{c}$, the ratio decreases from $\Delta a / \tilde{a}=\infty$ to $\Delta a / \tilde{a}=0$ at $\tilde{\xi}_{c}=\xi_{c}$, crossing 1 at $\tilde{\xi}_{c}=$ $\tilde{\xi}_{c}^{\ominus+}$. Here, $\left.\tilde{\xi}_{c}^{\ominus-} \in\right] 0.778,1\left[\right.$ and $\left.\tilde{\xi}_{c}^{\ominus-} \in\right] 1,1.284[$ are given by Eqs. (12) and (13) with $\eta_{c}\left(\xi_{c}, \Phi, l\right)=1+\frac{1}{2 \pi} \arctan \left(\frac{|a| \pi}{L}\right)$ where $a$ is the bare scattering length in Eq. (9). For the electronic density $n_{e}=0.176$ and interaction $u=U / 4 t=0.30$ [the values used in $\mathrm{Sec} . \mathrm{V}$ for $\mathrm{Bi} / \mathrm{InSb}(001)$ ], $\tilde{\xi}_{c}^{\ominus}=1 / \xi_{c}=$ $0.805, \tilde{\xi}_{c}^{\ominus-}=0.857$, and $\tilde{\xi}_{c}^{\ominus+}=1.166$.

The renormalized charge parameter intervals $\left.\tilde{\xi}_{c} \in\right] 1 / 2,1[$ for which $\alpha>1 / 8$ and $\left.\tilde{\xi}_{c} \in\right] 1, \xi_{c}$ ] for which $\alpha<1 / 8$ refer to two qualitatively different problems. Importantly, the $\tilde{\xi}_{c}$ value in the $\xi_{c} \rightarrow \tilde{\xi}_{c}$ transformation is uniquely defined for each of these two intervals solely by the bare charge parameter $\xi_{c}=\xi_{c}\left(n_{e}, u\right)$, Eq. (A16) of Appendix A, the integer quantum number $l$ in the potential $V_{c}(x)$ large- $x$ expression, Eq. (5), and its sum rule zero-energy phase $\Phi$, Eq. (7), as follows:

$$
\begin{aligned}
\tilde{\xi}_{c} & \left.=\eta_{c}\left(\xi_{c}, \Phi, l\right)\left(1-\sqrt{1-\frac{1}{\eta_{c}^{2}\left(\xi_{c}, \Phi, l\right)}}\right) \in\right] 1 / 2,1[ \\
& \left.\left.=\eta_{c}\left(\xi_{c}, \Phi, l\right)\left(1+\sqrt{1-\frac{1}{\eta_{c}^{2}\left(\xi_{c}, \Phi, l\right)}}\right) \in\right] 1, \xi_{c}\right],
\end{aligned}
$$

where

$$
\eta_{c}\left(\xi_{c}, \Phi, l\right)=1+\frac{1}{2 \pi} \arctan \left(\frac{\tan \left(\frac{\left(\xi_{c}-1\right)^{2} \pi}{\xi_{c}}\right)}{1+\tan \left(\frac{\pi}{l-2}\right) \tan (\Phi)}\right) .
$$

\section{THE EFFECTIVE RANGE}

\section{A. The effective-range general problem and cancellation of its unwanted terms}

The MQIM-HO accounts for the higher terms in the effective range expansion, Eq. (4), so that as anticipated the phase shift $2 \pi \tilde{\Phi}_{c, c}\left( \pm 2 k_{F}, q\right)$ acquires an additional term, $2 \pi \tilde{\Phi}_{c, c}^{R_{\text {eff }}}\left(k_{r}\right)$, relative to the MQIM-LO,

$$
\begin{aligned}
2 \pi \tilde{\Phi}_{c, c}\left( \pm 2 k_{F}, q\right)= & 2 \pi \tilde{\Phi}_{c, c}^{\tilde{a}}\left( \pm 2 k_{F}, q\right)+2 \pi \tilde{\Phi}_{c, c}^{R_{\text {eff }}}\left(k_{r}\right) \\
2 \pi \tilde{\Phi}_{c, c}^{\tilde{a}}\left( \pm 2 k_{F}, q\right)= & \frac{\xi_{c}}{\tilde{\xi}_{c}} \frac{\left(\tilde{\xi}_{c}-1\right)^{2}}{\left(\xi_{c}-1\right)^{2}} 2 \pi \Phi_{c, c}\left( \pm 2 k_{F}, q\right) \\
= & \frac{\arctan \left(\frac{\tilde{a}}{L} 2 \pi\right)}{\arctan \left(\frac{a}{L} 2 \pi\right)} 2 \pi \Phi_{c, c}\left( \pm 2 k_{F}, q\right), \\
2 \pi \tilde{\Phi}_{c, c}^{R_{\text {eff }}}\left(k_{r}\right)= & \arctan \left(\frac{1}{2} R_{\mathrm{eff}} k_{r} \sin ^{2}\right. \\
& \left.\times\left(\frac{\left(\tilde{\xi}_{c}-1\right)^{2}}{\tilde{\xi}_{c}} \pi\right)+P_{c}\left(k_{r}\right)\right) .
\end{aligned}
$$

The second term in the expression for the phase shift $2 \pi \tilde{\Phi}_{c, c}^{\tilde{a}}\left( \pm 2 k_{F}, q\right)$ reveals that its renormalization is controlled by the scattering lengths associated with the leading term in the effective range expansion. The $\tilde{\xi}_{c}=\xi_{c}$ bare phase shift $2 \pi \Phi_{c, c}\left( \pm 2 k_{F}, q\right)$ in that expression is defined in Eqs. (A11)(A15) of Appendix A. Furthermore, the function $P_{c}\left(k_{r}\right)$ in the expression of $2 \pi \tilde{\Phi}_{c, c}^{R_{\text {eff }}}\left(k_{r}\right)$ vanishes for $l<8$ and is such that its use in the term on the left-hand side of Eq. (4), $\cot \left(\tilde{\Phi}_{c}\left(k_{r}\right)\right)=\cot \left(-2 \pi \tilde{\Phi}_{c, c}^{\tilde{a}}\left( \pm 2 k_{F}, q\right)-2 \pi \tilde{\Phi}_{c, c}^{R_{\text {eff }}}\left(k_{r}\right)\right)$, gives rise to all the shape parameter terms in the expansion, Eq. (4), beyond the two leading terms, $\frac{-1}{\tilde{a} k_{r}}+\frac{1}{2} R_{\text {eff }} k_{r}$.

Fortunately, in the unitary limit all properties that are characterized by these higher-order terms become irrelevant also for $l>7$. Hence $2 \pi \widetilde{\Phi}_{c, c}^{R_{\text {eff }}}\left(k_{r}\right)$ is given by $\arctan \left(\frac{1}{2} R_{\text {eff }} k_{r}\right.$ $\left.\sin ^{2}\left(\left[\left(\tilde{\xi}_{c}-1\right)^{2} / \tilde{\xi}_{c}\right] \pi\right)\right)$, which gives $\cot \left(\tilde{\Phi}_{c}\left(k_{r}\right)\right)=\frac{-1}{\tilde{a} k_{r}}+$ $\frac{1}{2} R_{\text {eff }} k_{r}$ at small $k_{r}$. (That $2 \pi \tilde{\Phi}_{c, c}^{R_{\text {eff }}}(\mp 2 \pi / L)$ vanishes in the thermodynamic limit confirms that at $k_{r}=\mp 2 \pi / L$ the phase shift $2 \pi \tilde{\Phi}_{c, c}\left( \pm 2 k_{F}, \pm 2 k_{F}+k_{r}\right)$ has the same value $\pm\left(\tilde{\xi}_{c}-1\right)^{2} \pi / \tilde{\xi}_{c}$, Eq. (8), as for the MQIM-LO). 
Both the unitary limit and the fact that for $\left.\tilde{\xi}_{c} \in\right] 1 / 2,1[$ the scattering energy of the residual interactions of the $c$ particles and $c$ impurity are much smaller than the depth $-V_{c}\left(x_{1}\right)$ of the potential $V_{c}(x)$ will play important roles in the following derivations of the effective range $R_{\text {eff }}$ in the expression of $2 \pi \tilde{\Phi}_{c, c}^{R_{\text {eff }}}\left(k_{r}\right)$, Eq. (14).

First, note that the phase shift term $-2 \pi \tilde{\Phi}_{c, c}^{\tilde{a}}\left( \pm 2 k_{F}\right.$, $\left.\pm 2 k_{F}+k_{r}\right)$ [see Eq. (14)] of $\tilde{\Phi}_{c}\left(k_{r}\right)=-2 \pi \tilde{\Phi}_{c, c}\left( \pm 2 k_{F}\right.$, $\left.\pm 2 k_{F}+k_{r}\right)$ in the effective range expansion, Eq. (4), contributes only to the leading term in that expansion, $\frac{-1}{\tilde{a} k_{k}}$. Thus it does not contribute to the effective range $R_{\text {eff }}$. Indeed, that phase shift term reads $\mp\left(\tilde{\xi}_{c}-1\right)^{2} \pi / \tilde{\xi}_{c}$, Eq. (8), at $k_{r}=$ $\mp 2 \pi / L$ whereas it vanishes at $k_{r}=0$, so that in the thermodynamic limit the derivative $-2 \pi \partial \tilde{\Phi}_{c, c}^{\tilde{a}}\left( \pm 2 k_{F}, \pm 2 k_{F}+\right.$ $\left.k_{r}\right) /\left.\partial k_{r}\right|_{k_{r}=0}$ is ill defined.

For a potential with large- $x$ behavior, $-C_{c} /\left(x / 2 r_{l}\right)^{l}$, Eq. (5), the effective range $R_{\text {eff }}$ in the phase shift term $2 \pi \tilde{\Phi}_{c, c}^{R_{\text {eff }}}\left(k_{r}\right)$ of Eq. (14) follows from standard scatteringtheory methods, and becomes [21-23]

$$
R_{\mathrm{eff}}=2 \int_{0}^{\infty} d x\left(\left(\psi_{c}^{0}(x)\right)^{2}-\left(\psi_{c}(x)\right)^{2}\right) .
$$

This integral converges provided that $l>5$.

The bare limit $\tilde{\xi}_{c}=\xi_{c}$ boundary condition $V_{c}(x)=0$ for all $x$ corresponds to the wave function $\psi_{c}^{0}(x)$ in Eq. (15). It is the zero-energy solution of the Schrödinger equation for the free motion,

$$
-\frac{1}{2 \mu} \frac{d^{2} \psi_{c}^{0}(x)}{d x^{2}}=0
$$

Here, $\mu$ is the reduced mass of the $c$ particle and $c$ impurity. The function $\psi_{c}^{0}(x)$ then has the form $\psi_{c}^{0}(x)=1-x / a$ for all $x \in[0, \infty]$.

In contrast, the wave function $\psi_{c}(x)$ in Eq. (15) is associated with the potential $V_{c}(x)$ induced by the potential $V_{e}(r)$ in Eq. (1). The former is associated with the interaction of the $c$ particle and $c$ impurity. That wave function is thus the solution of a corresponding Schrödinger equation at zero energy,

$$
-\frac{1}{2 \mu} \frac{d^{2} \psi_{c}(x)}{d x^{2}}+V_{c}(x) \psi_{c}(x)=0,
$$

with the boundary condition $\psi_{c}(0)=0$. It is normalized at $x \rightarrow \infty$ as $\psi_{c}(x)=\psi_{c}^{0}(x)=1-x / a$.

The charge parameter interval $\left.\tilde{\xi}_{c} \in\right] 1, \xi_{c}$ ] for which $\alpha<$ $1 / 8$ that corresponds to the second $\tilde{\xi}_{c}$ expression in Eq. (12) is of little interest for our studies, as similar $\alpha$ values are reachable by the 1D Hubbard model. Two boundary conditions that must be obeyed by $R_{\text {eff }}$ in that parameter interval are $\lim _{\tilde{\xi}_{c} \rightarrow \xi_{c}} R_{\text {eff }}=0$ and $\lim _{\tilde{\xi}_{c} \rightarrow 1} R_{\text {eff }}=a_{0}$. They are satisfied by the following phenomenological effective range expression:

$$
R_{\mathrm{eff}}=a_{0}\left(1-\frac{\tilde{a}}{a}\right)<a_{0} .
$$

In the case of the interval $\left.\tilde{\xi}_{c} \in\right] 1 / 2,1$ [ for which $\alpha>1 / 8$ that corresponds to the first $\tilde{\xi}_{c}$ expression in Eq. (12), the explicit derivation of the integral in the effective range expression, Eq. (15), simplifies because the inequalities $\sqrt{2 \mu \gamma_{c}} \gg$ 1 in units of $a_{0}=1$ and $\Phi / \pi \gg 1$ are found to apply, as reported in Sec. III. This ensures that for $\tilde{\xi}_{c}<1$ the scattering energy of the residual interactions of the $c$ particles and $c$ impurity is much smaller than the depth $-V_{c}\left(x_{1}\right)$ of the potential $V_{c}(x)$.

The following analysis applies to general scattering lengths $a$, finite or infinite, and potentials with these general properties. They imply that the large- $x$ function $\psi_{c}(x)$ obeying a Schrödinger equation,

$$
\frac{d^{2} \psi_{c}(x)}{d x^{2}}+\frac{2\left(2 r_{l}\right)^{l-2}}{x^{l}} \psi_{c}(x)=0,
$$

whose attractive potential is given by its large-distance asymptotic behavior $V_{c}^{\text {asy }}(x)=-C_{c} /\left(x / 2 r_{l}\right)^{l}$, Eq. (5), which has the general form,

$$
\psi_{c}(x)=\sqrt{x}\left(B_{1} \phi_{\frac{1}{l-2}}(x)-B_{2} \frac{\phi_{\frac{-1}{l-2}}(x)}{\cos \left(\frac{\pi}{l-2}\right)}\right) .
$$

This expression can be used for all $x \in[0, \infty]$ provided that $V_{c}(x)$ at small distances $x \approx x_{1}$ where it is deep is replaced by a suitable energy-independent boundary condition. This is also valid for 3D s-wave scattering problems whose potentials have the above general properties and whose scattering lengths are parametrically large [23].

$B_{1}$ and $B_{2}$ are in Eq. (18) $x$ independent constants and $\phi_{\frac{1}{l-2}}(x)=J_{\frac{1}{l-2}}(y)$ and $\phi_{\frac{-1}{l-2}}(x)=J_{\frac{-1}{l-2}}(y)$ where $J_{\frac{1}{l-2}}(y)$ and $J_{\frac{-1}{l-2}}(y)$ are Bessel functions of argument,

$$
y=\frac{2 \sqrt{2}}{(l-2)\left(x / 2 r_{l}\right)^{\frac{l-2}{2}}} .
$$

From the use in Eq. (18) of the asymptotic behavior, $J_{v}(y) \approx$ $y^{v} /\left(2^{v} \Gamma(1+v)\right)$, of the Bessel functions for $x \gg 1$ and thus $y \ll 1$ one finds that the normalization at $x \rightarrow \infty$ as $\psi_{c}(x)=$ $\psi_{c}^{0}(x)=1-x / a$ requires that

$$
B_{1}=\frac{1}{\sqrt{2 r_{l}}}\left(\frac{l-2}{\sqrt{2}}\right)^{\frac{1}{l-2}} \Gamma\left(\frac{l-1}{l-2}\right)
$$

and

$$
\begin{aligned}
B_{2} & =B_{2}^{0}=\frac{\bar{a}}{a} B_{1}, \quad \text { where the length } \bar{a} \text { reads } \\
\bar{a} & =2 r_{l}\left(\frac{\sqrt{2}}{l-2}\right)^{\frac{2}{l-2}} \frac{\Gamma\left(\frac{l-3}{l-2}\right)}{\Gamma\left(\frac{l-1}{l-2}\right)} \cos \left(\frac{\pi}{l-2}\right) .
\end{aligned}
$$

It is convenient to write the integrand in Eq. (15) as $\left(\psi_{c}^{0}(x)\right)^{2}-\psi_{c}^{2}(x)=g_{c}^{\text {virtual }}(x)+g_{c}(x)$, where

$$
\begin{aligned}
g_{c}^{\text {virtual }}(x) & =\left(\psi_{c}^{0}(x)\right)^{2}-f_{c}(x) \text { and } \\
\psi_{c}(x) & =\sqrt{f_{c}(x)-g_{c}(x)},
\end{aligned}
$$

and the functions $f_{c}(x)$ and $g_{c}(x)$ are given by

$$
\begin{aligned}
f_{c}(x)= & \left(2 r_{l}\right)^{2} \frac{d}{d x}\left(( \frac { x } { 2 r _ { l } } ) ^ { 2 } \left\{B_{1}^{2} \phi_{\frac{1}{l-2}}^{2}(x)-\frac{B_{2}}{\cos \left(\frac{\pi}{l-2}\right)}\right.\right. \\
& \left.\left.\times\left[B_{1} \phi_{\frac{1}{l-2}}(x)-\frac{B_{2}}{3 \cos \left(\frac{\pi}{l-2}\right)} \phi_{\frac{-1}{l-2}}(x)\right] \phi_{\frac{-1}{l-2}}(x)\right\}\right)
\end{aligned}
$$


and

$$
\begin{aligned}
g_{c}(x)= & \left(\frac{x}{2 r_{l}}\right)^{-\frac{(l-2)}{2}+1} 4 \sqrt{2} r_{l}\left\{B_{1}^{2} \phi_{\frac{1}{l-2}}(x) \phi_{\frac{1}{l-2}+1}(x)\right. \\
& -\frac{B_{1} B_{2}}{2 \cos \left(\frac{\pi}{l-2}\right)}\left[\phi_{\frac{1}{l-2}}(x) \phi_{\frac{-1}{l-2}+1}(x)+\phi_{\frac{-1}{l-2}}(x) \phi_{\frac{1}{l-2}+1}(x)\right] \\
& \left.+\frac{B_{2}^{2}}{3 \cos ^{2}\left(\frac{\pi}{l-2}\right)} \phi_{\frac{-1}{l-2}}(x) \phi_{\frac{-1}{l-2}+1}(x)\right\}
\end{aligned}
$$

respectively.

The separation in Eq. (21) is convenient because the divergences all appear in the functions $\left(\psi_{c}^{0}(x)\right)^{2}$ and $f_{c}(x)$. That $B_{1}$ and $B_{2}$ have the expressions given in Eqs. (19) and (20), respectively, ensures that both $2 \int_{0}^{\infty} d x\left(\psi_{c}^{0}(x)\right)^{2}$ and $2 \int_{0}^{\infty} d x f_{c}(x)$ read $\lim _{x \rightarrow \infty} 2\left(x-\frac{x^{2}}{a}+\frac{1}{3} \frac{x^{3}}{a^{2}}\right)$ and thus the divergences from $\left(\psi_{c}^{0}(r)\right)^{2}$ and $f_{c}(x)$ exactly cancel each other under the integration of Eq. (15). Hence $R_{\text {eff }}$ can be expressed as

$$
R_{\mathrm{eff}}=2 \int_{0}^{\infty} d x g_{c}(x)
$$

\section{B. The energy-independent boundary condition}

The only role of $f_{c}(x)$, Eq. $(22)$, is to cancel $\left(\psi_{c}^{0}(x)\right)^{2}$ within $g_{c}^{\text {virtual }}(x)$, Eq. (21), under the integration in Eq. (15). In the general expression for the effective range given in that equation, $\psi_{c}(x)$ is the solution of Eq. (16) with the actual potential $V_{c}(x)$ defined in its full domain, $x \in[0, \infty]$. The alternative use of Eq. (24), which was derived by using the function $\psi_{c}(x)$ large- $x$ expression, Eq. (18), for the whole domain $x \in[0, \infty]$, also leads to the effective range, Eq. (15). This applies provided that $V_{c}(x)$ is replaced at small distances near $x=x_{1}$, where it is deep, by the energy-independent boundary condition defined below. It accounts for the effects from $V_{c}(x)$ for small distances.

In the unitary limit the inverse scattering length, $a^{-1}=$ 0 , which appears in the $B_{2}$ expression, Eq. (20), is at the middle of negative $a^{-1}<0$ and positive $a^{-1}>0$ values and could refer to $a=-\infty$ or $a=\infty$. Hence in that limit there is not much difference between the repulsive and attractive scattering cases. As discussed in Ref. [41] for the case of two particles with a s-wave interaction, the scattering lengths in the attractive $a=-\infty$ and repulsive $a=\infty$ cases of the unitary limit merely refer to different states of the same $a^{-1}=$ 0 scattering problem.

For a potential $V(r)$ with a finite scattering length $a$ and having the general properties reported above, at small distances $r$ where the potential is deep it can be replaced by an energy-independent boundary condition such that the ratio $B_{2} / B_{1}=\bar{a} / a$ reads $\left[1-\tan \left(\frac{\pi}{l-2}\right) \tan (\bar{\Phi})\right]^{-1}$ where $\bar{\Phi}=$ $\int_{r_{0}}^{\infty} d r \sqrt{2 \mu(-V(r))}-\pi /[2(l-2)]$. Here, $\bar{\Phi} \gg \pi$ is a potential dependent zero-energy phase, $V\left(r_{0}\right)=0$, and $V(r)<0$ for $r>r_{0}$. Moreover, $\tan (\bar{\Phi})=-\frac{\Delta a}{\bar{a}} \cos \left(\frac{\pi}{l-2}\right)$ where $\Delta a=$ $a-\bar{a}, \Delta a / \bar{a}$ is a relative fluctuation, and $\bar{a}$ given in Eq. (20) is a mean scattering length determined by the asymptotic behavior $\propto 1 / r^{l}$ of the potential $V(r)$ through the integer $l>5$ and the length scale $2 r_{l}$. For instance, in terms of the constants $A=B_{1}-B_{2}$ and $B=-B_{2} \tan \left(\frac{\pi}{l-2}\right)$, of the scattering problem studied in Ref. [23], the ratio $\bar{a} / a=B_{2} / B_{1}$ on the left-hand side of the above boundary condition reads $\left[1-(A / B) \tan \left(\frac{\pi}{n-2}\right)\right]^{-1}$ where $n=l$.

For the present range $\left.\tilde{\xi}_{c} \in\right] 1 / 2,1$ [ the length scale $2 r_{l}$ whose expression is given below is finite in the unitary limit and thus the related length scale $\bar{a}$ in Eq. (20) is also finite. It follows both that $\bar{a} / a=0$ and the constant $B_{2}=B_{2}^{0}$, Eq. (20), vanishes. This result is clearly incorrect. The reason is that we have have not yet accounted for the behavior of $V_{c}(x)$ at small distances through a suitable energy-independent boundary condition. In the case of the unitary limit, this boundary condition renders both $\bar{a} / a$ and $B_{2}=B_{2}^{0}$ in Eq. (20) finite. Specifically, the scattering length $a$ is suitably mapped under it into a finite scattering length $a_{\mathrm{f}}=a \frac{\bar{a}}{\tilde{a}}$, so that $B_{2}^{0}$ is mapped onto the following corresponding finite constant $B_{2}$ :

$$
\begin{aligned}
& B_{2}=\frac{\bar{a}}{a_{\mathrm{f}}} B_{1}=\frac{\tilde{a}}{a} B_{1}, \text { where } \\
& a_{\mathrm{f}}=a \frac{\bar{a}}{\tilde{a}}=\bar{a}\left[1-\tan \left(\frac{\pi}{l-2}\right) \tan \left(\bar{\Phi}_{\mathrm{f}}\right)\right] .
\end{aligned}
$$

Here $\tan \left(\bar{\Phi}_{\mathrm{f}}\right)=\tan (\Phi)$ yet $\bar{\Phi}_{\mathrm{f}} \gg \pi$ may be different from $\Phi \gg \pi$ in Eq. (10). Indeed, the relation $\tan \left(\bar{\Phi}_{\mathrm{f}}\right)=\tan (\Phi)$ is insensitive to such phase differences. In the unitary limit the boundary condition is thus equivalent to a transformation $a \rightarrow a_{\mathrm{f}}$ such that $\tan \left(\bar{\Phi}_{\mathrm{f}}\right)=\tan (\Phi)$.

The energy-independent boundary condition in Eq. (25) is in terms of the finite scattering length $a_{\mathrm{f}}$ such that $B_{2} / B_{1}=$ $\bar{a} / a_{\mathrm{f}}$ is given by $\left[1-\tan \left(\frac{\pi}{l-2}\right) \tan \left(\bar{\Phi}_{\mathrm{f}}\right)\right]^{-1}$, similarly to scattering problems of the same universality class whose scattering lengths are parametrically large $[23,32]$. The positivity of $a_{\mathrm{f}}=a \frac{\bar{a}}{\tilde{a}}$ often occurs for potentials that for large distances are attractive [23]. If $a_{\mathrm{f}}$ were negative, $\bar{a} / a_{\mathrm{f}}=-\tilde{a} / a$, then $\tan \left(\bar{\Phi}_{\mathrm{f}}\right)$ would be given by $2 \cot \left(\frac{\pi}{l-2}\right)-\tan (\Phi)$, which would violate both the requirements that $\tan \left(\bar{\Phi}_{\mathrm{f}}\right)=\tan (\Phi)$ and that $\tan \left(\bar{\Phi}_{\mathrm{f}}\right)=0$ in the bare limit, $\tilde{\xi}_{c}=\xi_{c}$.

Importantly, the cancellation $\left(\psi_{c}^{0}(x)\right)^{2}-f_{c}(x)=0$ is independent of the value of the scattering length in the expressions for $\psi_{c}^{0}(x)$ and $f_{c}(x)$. Hence all results associated with Eqs. (15)-(24) remain the same, with $a$ replaced by $a_{\mathrm{f}}$. This includes the effective range $R_{\text {eff }}$, Eq. (24), remaining determined only by $g_{c}(x)$.

The main property of the transformation $a \rightarrow a_{\mathrm{f}}$ is the corresponding exact equality of the ratios, $\bar{a} / a_{\mathrm{f}}=\tilde{a} / a$. This actually justifies why the scattering lengths $a$ and $\tilde{a}$, Eqs. (9), can be used in $\tan (\Phi)$ in Eq. (6). That transformation is also the mechanism through which the renormalized scattering length $\tilde{a}$ emerges in $\psi_{c}(x)$.

Hence similarly to finite- $a$ scattering problems of the same universality class, as for instance those studied in Refs. [23,37], the relations of general form, Eq. (6), apply. In the present unitary limit, the scattering length ratio $\tilde{a} / a$ in them equals the ratio $\bar{a} / a_{\mathrm{f}}$ also given by Eq. (10). The sum rule $\Phi=\int_{x_{0}}^{x_{2}} d x \sqrt{2 \mu\left(-V_{c}(x)\right)}$, Eq. (7), encodes the effects from $V_{c}(x)$ for small distance near $x=x_{1}$, referring to the interval $x \in\left[x_{0}, x_{2}\right]$ around $x_{1}$. 
TABLE II. Length scales involved in the MQIM-HO description.

\begin{tabular}{lc}
\hline \hline Length scale & Description \\
\hline $2 r_{l}$ & length scale in the large- $x$ potential decay with exponent $l>5, V_{c}^{\text {asy }}(x) \propto\left(x / 2 r_{l}\right)^{-l}$, Eqs. $(5)$ and $(29)$ \\
$a_{0}$ & lattice spacing related to $2 r_{l}$ (twice the van der Waals length at $\left.l=6\right)$ as given in Eq. (27) \\
$a$ and $\tilde{a}$ & scattering lengths at $\xi_{c}$ and $\tilde{\xi}_{c}$, respectively, within the $\xi_{c} \rightarrow \tilde{\xi}_{c}$ transformation, Eqs. (9) and (10) \\
$R_{\text {eff }}$ & effective range $R_{\text {eff }}=a_{0}\left(1-c_{1}\left(\frac{\tilde{a}}{a}\right)+c_{2}\left(\frac{\tilde{a}}{a}\right)^{2}\right)$ for the interval $\tilde{\xi}_{c}<1$ of physical interest, Eqs. (26) and (28) \\
\hline \hline
\end{tabular}

\section{The effective range dependence on the scattering length finite ratio $\tilde{a} / a$}

The use of the function $g_{c}(x)$, Eq. (23), with the constants $B_{1}$ and $B_{2}$ given in Eqs. (19) and (25), respectively, in Eq. (24) leads for $\left.\widetilde{\xi}_{c} \in\right] 1 / 2,1[$ to

$$
\begin{aligned}
R_{\mathrm{eff}}= & 2 \sqrt{2} \Gamma^{2}\left(\frac{l-1}{l-2}\right)\left(\frac{l-2}{\sqrt{2}}\right)^{\frac{2}{l-2}} \\
& \times\left[\int_{0}^{\infty} d x\left(\frac{x}{2 r_{l}}\right)^{-\frac{l-2}{2}+1} \phi_{\frac{1}{l-2}}(x) \phi_{\frac{-1}{l-2}+1}(x)\right. \\
& -\left(\frac{\tilde{a}}{a}\right) \int_{0}^{\infty} d x\left(\frac{x}{2 r_{l}}\right)^{-\frac{l-2}{2}+1} \\
& \times \frac{\phi_{\frac{1}{l-2}}(x) \phi_{\frac{-1}{l-2}+1}(x)+\phi_{\frac{-1}{l-2}}(x) \phi_{\frac{1}{l-2}+1}(x)}{2 \cos \left(\frac{\pi}{l-2}\right)} \\
& \left.+\left(\frac{\tilde{a}}{a}\right)^{2} \int_{0}^{\infty} d x\left(\frac{x}{2 r_{l}}\right)^{-\frac{l-2}{2}+1} \frac{\phi_{\frac{-1}{l-2}}(x) \phi_{\frac{-1}{l-2}+1}(x)}{3 \cos ^{2}\left(\frac{\pi}{l-2}\right)}\right] .
\end{aligned}
$$

After performing the integrations, one finally reaches the following expression valid for the present charge parameter interval $\left.\widetilde{\xi}_{c} \in\right] 1 / 2,1[$ and $\alpha>1 / 8$ :

$$
R_{\mathrm{eff}}=a_{0}\left[1-c_{1}\left(\frac{\tilde{a}}{a}\right)+c_{2}\left(\frac{\tilde{a}}{a}\right)^{2}\right] .
$$

That here the coefficient

$$
a_{0}=2 r_{l}\left(\frac{2}{3 \pi} \frac{\left(\frac{2}{(l-2)^{2}}\right)^{\frac{1}{l-2}}}{\sin \left(\frac{\pi}{l-2}\right)}\right) \frac{\Gamma\left(\frac{1}{l-2}\right) \Gamma\left(\frac{4}{l-2}\right)}{\Gamma^{2}\left(\frac{2}{l-2}\right) \Gamma\left(\frac{3}{l-2}\right)}
$$

is identified with the lattice spacing results from the imposition of $R_{\text {eff }}$ having the same value for $\tilde{\xi}_{c} \rightarrow 1^{-}$and $\tilde{\xi}_{c} \rightarrow 1^{+}$. The coefficients $c_{1}$ and $c_{2}$ in Eq. (26) can be expressed in terms the usual $\Gamma$ function and are given by

$$
\begin{aligned}
& c_{1}=\frac{2}{\cos \left(\frac{\pi}{l-2}\right)} \frac{\Gamma\left(\frac{2}{l-2}\right) \Gamma\left(\frac{l-4}{l-2}\right)}{\Gamma\left(\frac{1}{l-2}\right) \Gamma\left(\frac{l-3}{l-2}\right)} \text { and } \\
& c_{2}=\frac{3(l+1)}{(l-1) \cos ^{2}\left(\frac{\pi}{l-2}\right)} \frac{\Gamma\left(\frac{3}{l-2}\right) \Gamma\left(-\frac{l+1}{l-2}\right)}{\Gamma\left(\frac{-1}{l-2}\right) \Gamma\left(-\frac{l-1}{l-2}\right)},
\end{aligned}
$$

respectively. They decrease from $c_{1}=c_{2}=2$ at $l=6$ to $c_{1}=$ 1 and $c_{2}=1 / 3$ for $l \rightarrow \infty$.

The effective range $R_{\text {eff }}$, Eq. (26), appears in the expression of the spectral function exponents $\tilde{\zeta}_{c}(k)$ and $\tilde{\zeta}_{c^{\prime}}(k)$, Eq. (A3) of Appendix A, through the expression for the phase shift $2 \pi \tilde{\Phi}_{c, c}\left( \pm 2 k_{F}, q\right)$, Eq. (14). $R_{\text {eff }}=\infty$ for $\tilde{\xi}_{c} \rightarrow 1 / 2$ is excluded, as it is outside the range of validity of the unitary limit. The $R_{\text {eff }}$ values found below in $\mathrm{Sec} . \mathrm{V}$ for $\mathrm{Bi} / \mathrm{InSb}(001)$ are given in Table $\mathrm{V}$. They obey the unitary limit inequality, $R_{\text {eff }} \ll 1 / k_{F c}^{0}$.

The effective range, Eq. (26), can alternatively be expressed in terms of the ratio $\bar{a} / a_{\mathrm{f}}$ involving the finite scattering lengths $\bar{a} \propto 2 r_{l}$ and $a_{\mathrm{f}}$ defined by Eqs. (20) and (25), respectively.

The expression for the lattice spacing $a_{0}$, Eq. (27), contains important physical information: Its inverse gives the following expression valid for $\left.\tilde{\xi}_{c} \in\right] 1 / 2,1$ [ for the length scale $2 r_{l}$ in the potential $V_{c}^{\text {asy }}(x)$ expression, Eq. (5), and the related length scale $x_{2}$, Eq. (7),

$$
2 r_{l}=\frac{3 \pi a_{0}}{2} \sin \left(\frac{\pi}{l-2}\right)\left(\frac{l-2}{\sqrt{2}}\right)^{\frac{2}{l-2}} \frac{\Gamma^{2}\left(\frac{2}{l-2}\right) \Gamma\left(\frac{3}{l-2}\right)}{\Gamma\left(\frac{1}{l-2}\right) \Gamma\left(\frac{4}{l-2}\right)} .
$$

Here $2 r_{l}$ is given by $5.95047 a_{0}$ at $l=6$, reaches a maximum $6.48960 a_{0}$ at $l=10$, and decreases to $4.93480 a_{0}$ as $l \rightarrow \infty$, so that $\sqrt{2}\left(2 r_{l}\right)^{\frac{l-2}{2}}=\sqrt{2 \mu \gamma_{c}} \gg 1$ in units of $a_{0}=1$ as given in Table V. Thus $\Phi / \pi \gg 1$ for $l=6-12$.

As in the case of 3D s-wave atomic scattering problems $[23,32]$, this shows that for $\left.\tilde{\xi}_{c} \in\right] 1 / 2,1$ [ the scattering energy of the interactions of the $c$ particles and $c$ impurity is indeed much smaller than the depth $-V_{c}\left(x_{1}\right)$ of the potential $V_{c}(x)$ well. This confirms the consistency of the derivation of the effective range for $\left.\tilde{\xi}_{c} \in\right] 1 / 2,1$ [ that assumed the validity of such properties. The length scales involved in the MQIM-HO description are explicitly defined in Table II.

\section{ARPES IN Bi/InSb(001)}

\section{A. Brief information on the sample preparation and ARPES experiments}

Concerning the preparation of the $\mathrm{Bi} / \mathrm{InSb}(001)$ surface, a substrate $\mathrm{InSb}(001)$ was cleaned by repeated cycles of $\mathrm{Ar}$ sputtering and annealing up to $680 \mathrm{~K}$. Bi was evaporated on it up to nominally 3 monolayers (ML): One ML is defined as the atom density of bulk-truncated substrate. Then, the substrate was flash-annealed up to $680 \mathrm{~K}$ for $\sim 10$ seconds. The resulting surface showed a $(1 \times 3)$ low-energy electron diffraction pattern.

Although the $\mathrm{Bi} / \mathrm{InSb}(001)$ surface state is formed by evaporating $\mathrm{Bi}$ on the $\mathrm{InSb}$ substrate, in addition to $\mathrm{Bi}$ also In and $\mathrm{Sb}$ are found at the surface, modified from their bulk positions by $\mathrm{Bi}$ evaporation. Hence $\mathrm{Bi}, \mathrm{In}$, and $\mathrm{Sb}$ can all be significant sources of the surface electronic states. Detailed information of the characterization of the $\mathrm{Bi} / \mathrm{InSb}(001)$ surface sample is provided in Ref. [17].

ARPES measurements were performed at $\hbar \omega=15 \mathrm{eV}$ and taken at $8 \mathrm{~K}$ in the CASSIOPÉE beamline of SOLEIL synchrotron. The photoelectron kinetic energy at $E_{F}$ and the overall energy resolution of the ARPES setup were calibrated 

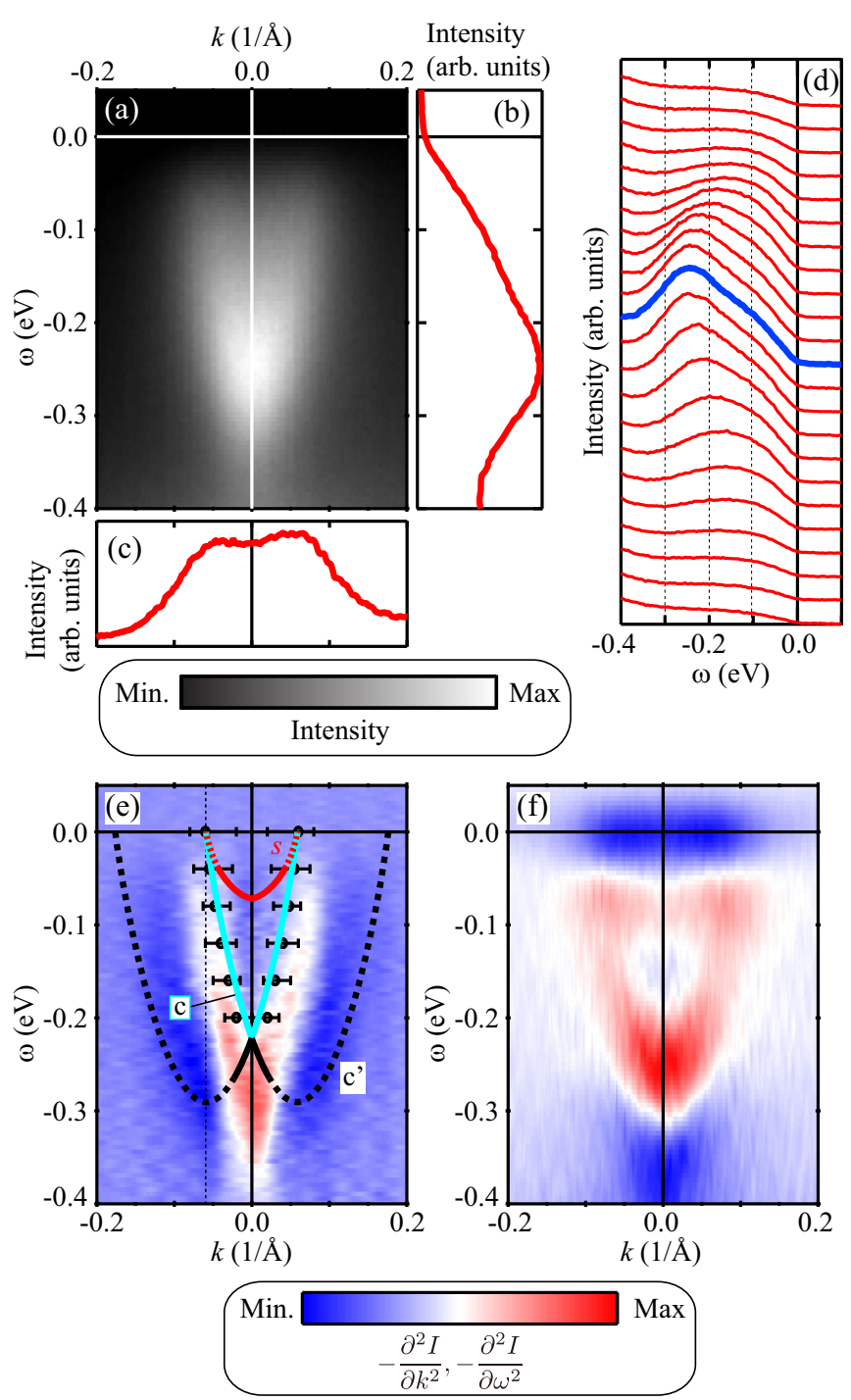

FIG. 3. (a) Raw Bi/InSb(001) ARPES data for $\hbar \omega=15 \mathrm{eV}$.

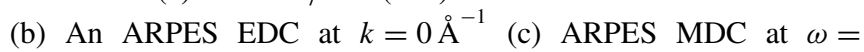
$-0.05 \mathrm{eV}$. (d) ARPES EDC from $k=-0.16$ (bottom) to +0.16 (top) $\AA^{-1}$. The thick line is the normal-emission spectrum $(k=$ $0 \AA^{-1}$ ). (e) and (f) Second-derivative ARPES images. Derivation was made along momentum in (e) and energy in (f). Circles and error bars in (e) indicate the MDC peak positions. Solid and dashed lines overlaid in (e) are the theoretical $s$ (red), $c$ (light blue), and $c^{\prime}$ (black) branch lines for $u=U / 4 t=0.30, t=1.22 \mathrm{eV}$, and electronic density $n_{e}=0.176$. Only for the solid-line $k$ ranges in (e) for which the exponents are negative in Fig. 4 and Figs. 5 and 6 of Appendix A can they be seen in the ARPES image. (ARPES from the same experimental data as in Refs. $[17,18])$.

by the Fermi edge of the photoelectron spectra from Mo foils attached to the sample. The energy resolution was $\sim 20 \mathrm{meV}$. The ARPES taken at $8 \mathrm{~K}$ is shown in Fig. 3.

The theoretical predictions reported in this paper refer to (i) the $(k, \omega)$-plane location of the high-energy $\mathrm{Bi} / \mathrm{InSb}(001)$ MDC and EDC ARPES peaks and (ii) the value of the powerlaw SDS exponent $\alpha$ associated with the angle integration to detect the low-energy suppression of the photoelectron intensity that were performed at $k_{y}=0.2 \AA^{-1}$, near the boundary of the $(1 \times 3)$ surface Brillouin zone $\left(0.23 \AA^{-1}\right)$.

\section{B. Criteria for agreement between ARPES and the present theory}

References [17,18] found strong experimental evidence that $\mathrm{Bi} / \operatorname{InSb}(001)$ at $y$ momentum component $k_{y}=0.2 \AA^{-1}$ and temperature $8 \mathrm{~K}$ displays $1 \mathrm{D}$ physics with an SDS exponent that for small $|\omega|<0.10 \mathrm{eV}$ has values in the interval $\alpha \in[0.6,0.7]$.

As discussed and justified below in Sec. VI A, the oneelectron spectral properties of $\mathrm{Bi} / \mathrm{InSb}(001)$ are expected to be controlled mainly by the interplay of one dimensionality and finite-range electron interactions, despite a likely small level of disorder. Consistent with an SDS exponent $\alpha$ larger than $1 / 8$ stemming from finite-range interactions [8], here we use the MQIM-HO to predict one-electron spectral properties of $\mathrm{Bi} / \mathrm{InSb}(001)$.

As discussed in $\mathrm{Sec}$. VI A, $\mathrm{Bi} / \mathrm{InSb}(001)$ is a complex system and some of its experimental properties beyond those studied here may involve microscopic processes other than those described by the MQIM-HO and the Hamiltonian, Eq. (1). This includes coupling to two-dimensional (2D) physics if $k_{y}=0.2 \AA^{-1}$ is smoothly changed to $k_{y}=0$.

As reported in Sec. III A, the MQIM-HO can describe both the low-energy TLL regime and the spectral function, Eq. (2), at high energies near the $(k, \omega)$-plane singularities. At and in the vicinity of those singularities, the renormalization from its bare $\tilde{\xi}_{c}=\xi_{c}$ form is determined by the large $x$ behavior of $V_{c}(x)$, Eq. (5), and its sum rules, Eqs. (6) and (7), which refer to a high-energy regime that goes well beyond the TLL limit.

Hence we can predict two properties of the one-electron spectral function : (i) the location in the $(k, \omega)$ plane of the experimentally observed high-energy peaks in the ARPES MDC and EDC and (ii) the value of the low-energy SDS exponent $\alpha$. Our $T=0$ theoretical results describe the former high-energy experimental data taken at $8 \mathrm{~K}$ for which the smearing of the spectral function singularities by thermal fluctuations is negligible. The quantitative agreement with the corresponding experimental data taken at fixed momentum $k_{y}=0.2 \AA^{-1}$ reached below provides further evidence of 1D physics and electron finite-range interactions in $\mathrm{Bi} / \mathrm{InSb}(001)$.

A first type of agreement of the theoretical branch-line energy spectra with the $(k, \omega)$-plane shape of the ARPES image spectra must be reached for well-defined fixed values of electronic density $n_{e}$ and interaction $u=U / 4 t$. Through Eq. (A16) of Appendix (A), these uniquely determine the value of the bare charge parameter $\xi_{c}=\xi_{c}\left(u, n_{e}\right)$ to be used in the $\xi_{c} \rightarrow \tilde{\xi}_{c}$ transformations suited to $\mathrm{Bi} / \mathrm{InSb}(001)$. In addition, that first type of agreement also determines the value of the transfer integral $t$.

The experimental values of the lattice spacing $a_{0}$ and of the momentum width of the spectra at $\omega=0$ provide the Fermi momentum $k_{F}=\left(\pi / 2 a_{0}\right) n_{e}$ and thus the electronic density $n_{e}$. At the density $n_{e}$, the ratio $\tilde{W}_{s} / \tilde{W}_{c}$ of the experimental energy bandwidths $\tilde{W}_{s} \equiv\left|\tilde{\omega}_{s}(0)\right|$ of the $s$ branch line spectrum and $\tilde{W}_{c} \equiv\left|\tilde{\omega}_{c}(0)\right|=\left|\tilde{\omega}_{c^{\prime}}(0)\right|$ of the $c$ and $c^{\prime}$ branch line spectra at momentum $k=0$ uniquely determine $u=U / 4 t$. (See such 
TABLE III. Types of agreement between theory and experiments.

\begin{tabular}{lc}
\hline \hline Agreement & Description \\
\hline First type & overall $(k, \omega)$-plane shapes of the theoretical branch-line spectra, Eq. (A1), versus ARPES experimental spectra \\
Second type & $(k, \omega)$-plane location of the singularities corresponding to negative exponents, Eq. (A3), versus ARPES peaks \\
Third type & SDS exponent $\alpha$ from the dependence of the exponents, Eq. (A3), on $\tilde{\xi}_{c}$ versus its low- $\omega$ experimental value \\
\hline \hline
\end{tabular}

energy bandwidths in the sketch of the theoretical spin $s$ and charge $c$ and $c^{\prime}$ branch lines in Fig. 1). Finally, the experimental values of $\tilde{W}_{s}$ and $\tilde{W}_{c}$ determine the value of the transfer integral $t$.

As discussed below in Sec. V C, from the available experimental data, it is not possible to trace the energy dispersion of the $s$ branch line. However, combining the experimental data on the EDC with kinematic constraints of the MDC provides information about the most probable value of the energy at which its bottom is located, which equals the branch line energy bandwidth $\tilde{W}_{s}$.

A second type of agreement is between the momentum interval and corresponding energy interval for which the exponents $\tilde{\zeta}_{c}(k), \tilde{\zeta}_{c^{\prime}}(k)$, and $\tilde{\zeta}_{s}(k)$, Eq. (A3) of Appendix A, are negative and the $(k, \omega)$-plane location of the experimentally observed high-energy ARPES MDC and EDC peaks. That agreement must be reached at the fixed $u$ and $n_{e}$ values and corresponding bare charge parameter $\xi_{c}=\xi_{c}\left(u, n_{e}\right)$ value obtained from the first type of agreement. This second type of agreement is reached at some values of the integer quantum number $l>5$ in the large- $x$ potential $V_{c}(x)$ expression, Eq. (5), and of the renormalized charge parameter $\tilde{\xi}_{c}$ [and thus of $\tan (\Phi)$, see Eqs. (12) and (13)].

For the theoretically predicted high-energy ARPES peaks located on the $s$ branch line, there is only limited experimental information. Hence we start by finding the $\tilde{\xi}_{c}$ and $l>5$ values at which the second type of agreement is reached concerning the momentum intervals where the exponents $\tilde{\zeta}_{c}(k)$ and $\tilde{\zeta}_{c^{\prime}}(k)$ are negative and the corresponding $(k, \omega)$-plane location of the experimentally observed high-energy ARPES MDC and EDC peaks. Fortunately, it turns out that the $\tilde{\xi}_{c}$ values lead to a prediction of location in the $(k, \omega)$ plane of the highenergy ARPES peaks associated with the $s$ branch line that is consistent with the available experimental EDC data.

This second type of agreement is reached for specific $\tilde{\xi}_{c}$ values. This then provides a prediction for the SDS exponent $\alpha=\left(2-\tilde{\xi}_{c}^{2}\right)^{2} /\left(8 \tilde{\xi}_{c}^{2}\right)$ obtained from a different low-energy experiment that detects the suppression of the photoelectron intensity. That the SDS exponent $\alpha$ determined by the $\tilde{\xi}_{c}$ values for which the second type of agreement is reached is also that measured within the low-energy angle integrated photoemission intensity then becomes the required third type of agreement.

In the Lehmann representation of the spectral function, the first and second types of agreement correspond to the energy spectra and the overlaps of the one-electron matrix elements, respectively. The exponents in Eq. (A3) of Appendix A involved in the second type of agreement depend both on $\tilde{\xi}_{c}$ and momentum-dependent phase shifts $\tilde{\Phi}_{c, c}\left( \pm 2 k_{F}, q\right)$ and $\tilde{\Phi}_{c, s}\left( \pm 2 k_{F}, q^{\prime}\right)$. There is no apparent direct relation between the high-energy ARPES MDC peaks and the low-energy SDS. That the MQIM-HO describes the main microscopic mechanisms behind the specific one-electron spectral properties of $\mathrm{Bi} / \mathrm{InSb}(001)$ then requires that the third type of agreement is fulfilled. The three types of agreement between theory and experiment are explicitly described in Table III.

\section{Searching for agreement between theory and experiments}

\section{First type of agreement}

The MDC spectral shape plotted in Fig. 3(c) displays two peaks centered at well defined Fermi momentum values $-k_{F}=-0.06 \AA^{-1}$ and $k_{F}=0.06 \AA^{-1}$, respectively. Furthermore, the experimental circles (with error bars) in Fig. 3(e) clearly indicate that the MDC peaks are located on two lines that in the limit of zero energy start at such two Fermi momenta. Since the experimental data lead to $\pi / a_{0} \approx 0.68 \AA^{-1}$, one finds from $k_{F}=\left(\pi / 2 a_{0}\right) n_{e} \approx 0.06 \AA^{-1}$ a small electronic density, $n_{e} \approx 0.176$.

The experimental value of the $c$ branch line energy bandwidth $\tilde{W}_{c}$ is directly extracted from analysis of the experimental MDC data provided in Fig. 3(e). From analysis of the EDCs in Fig. 3(d) alone one finds that there is a uncertainty $0.05 \pm 0.05 \mathrm{eV}$ concerning the energy at which the bottom of the $s$ branch line is located. It is clear that in this energy region there is a hump that cannot be explained by assuming the single peak at $0.25 \mathrm{eV}$, which refers to the bottom of the $c$ branch line.

The zero-energy level of the theoretically predicted downward-convex parabolic-like dispersion of the $s$ branch line plotted in Fig. 3(e) (see also sketch depicted in Fig. 1) refers to the Fermi level. Hence the $s$ branch line energy bandwidth $\tilde{W}_{s}$ equals that of its bottom. While the energy range uncertainty of that bottom energy is experimentally rather wide, one can lessen it by combining the experimental ARPES MDC intensity distribution shown in Fig. 3(c) with its kinematical constraints, which follow from the finite-energy bandwidth of the theoretical $s$ branch line. One then finds that the most probable value of the $s$ branch line bottom energy and thus of $\tilde{W}_{s}$ is between 0.05 and $0.10 \mathrm{eV}$.

The maximum momentum width of the ARPES MDC intensity distribution shown in Fig. 3(c) for energy $|\omega|=$ $0.05 \mathrm{eV}$ allowed by such kinematic constraints involves the superposition of two maximum momentum widths $\Delta k$, centered at $-k_{F}$ and $k_{F}$, respectively. Within the MQIM-HO, these kinematical constraints explain the lack of spectral weight in well-defined $(k, \omega)$-plane regions shown in Fig. 1. Fortunately, the lines that limit such regions without spectral weight only involve the $s$ band dispersion spectrum.

In the case of the spectral weight centered at $-k_{F}$ and $k_{F}$, respectively, such kinematical constraints imply that for each energy value $|\omega|=-\omega$ the corresponding maximum 
momentum width reads

$$
\begin{aligned}
\Delta k & \left.=2\left(k_{F}-k\right) \text { for }|\omega|=\left|\tilde{\omega}_{s}(k)\right| \text { and } k \in\right] 0, k_{F}[ \\
& \left.=2\left(k_{F}+k\right) \text { for }|\omega|=\left|\tilde{\omega}_{s}(k)\right| \text { and } k \in\right]-k_{F}, 0[
\end{aligned}
$$$$
\text { no constraints for }|\omega|>\tilde{W}_{s} \text { and }|k|>k_{F} \text {, }
$$

where $\tilde{W}_{s}=\left|\tilde{\omega}_{s}(0)\right|$ and the $s$ band dispersion spectrum $\tilde{\omega}_{s}(k)$ is given in Eq. (A1) of Appendix A.

For $|\omega| \ll \tilde{W}_{s}$ the kinematical constraints, Eq. (30), are those of a TLL, $\Delta k=2|\omega| / v_{s}\left(k_{F}\right)$, consistent with $v_{s}\left(k_{F}\right)=$ $\min \left(v_{s}\left(k_{F}\right), v_{c}\left(2 k_{F}\right)\right)$ [33]. However, for energy $|\omega|=-\omega$ larger than the $s$ branch line energy bandwidth $\tilde{W}_{s}=\left|\tilde{\omega}_{s}(0)\right|$, which is that at which the $s$ branch line bottom is located in the experimental data, there are no kinematical constraints.

The absolute value of the derivative with respect to $k$ of the ARPES MDC intensity plotted in Fig. 3(c) increases in a $|k|$ interval $|k| \in\left[k_{F}, k_{F}+k_{\mathrm{MDC}}\right]$ and decreases for $|k|>$ $k_{\mathrm{MDC}}$. Theoretically, the ARPES MDC intensity should be symmetrical around $k=0$. Its actual experimental shape then introduces a small uncertainty in the value of $k_{\mathrm{MDC}}$. The relatively large intensity in the tails located at the momentum region $|k|>k_{\mathrm{MDC}}$ is explained by the larger uncertainty in the $s$ branch line bottom energy $\tilde{W}_{s}$. Indeed, the ARPES MDC under consideration refers to an energy $|\omega|=0.05 \mathrm{eV}$ within that uncertainty. And, as given in Eq. (30), there are no kinematic constraints for $|\omega|>\tilde{W}_{s}$.

One can then identify the most probable value of $\tilde{W}_{s}$ within its uncertainty interval as that for which at the energy $|\omega|=$ $0.05 \mathrm{eV}$ the kinematic constraints would limit the ARPES MDC intensity to momentum values within the interval $|k| \leqslant$ $k_{\mathrm{MDC}}$. The corresponding momenta $k= \pm k_{\mathrm{MDC}}$ are the inflection points at which the derivative of the variation of the ARPES MDC intensity with respect to $k$ changes sign in Fig. 3(c). The momentum width associated with $|k| \leqslant k_{\mathrm{MDC}}$ is thus that of the ARPES MDC shown in that figure if one excludes the tails.

The corresponding maximum momentum width $\Delta k$, Eq. (30), of the two overlapping spectral weights centered at $k_{F}$ and $-k_{F}$, respectively, that at $|\omega|=0.05 \mathrm{eV}$ would lead to the kinematic constraint $\Delta k=2\left(k_{\mathrm{MDC}}-k_{F}\right)$, so that $\pm\left(k_{F}+\Delta k / 2\right)= \pm k_{\mathrm{MDC}}$. According to the kinematic constraints in Eq. (30), this is fulfilled when at $k= \pm\left(k_{F}-\right.$ $\Delta k / 2)= \pm\left(2 k_{F}-k_{\mathrm{MDC}}\right)$ so that the $s$ branch line energy spectrum reads $\left|\tilde{\omega}_{s}(k)\right|=-\tilde{\omega}_{s}(k)=0.05 \mathrm{eV}$. Accounting for the combined $k_{\mathrm{MDC}}$ and $\tilde{W}_{s}$ uncertainties, the most probable value of the energy bandwidth $\tilde{W}_{s}$ is larger than $0.05 \mathrm{eV}$ and smaller than $0.10 \mathrm{eV}$, as that of the theoretical $s$ branch line plotted in Fig. 3(e).

At electronic density $n_{e}=0.176$, the best second type of agreement between theory and experiments discussed in the following is reached within that combined uncertainty by the $u=U / 4 t$ and $t$ values that are associated with the energy bandwidth $\tilde{W}_{s}$ of such a theoretical $s$ branch line. They read $u=0.30$ and $t=1.22 \mathrm{eV}$, as determined from the corresponding ratio $\tilde{W}_{s} / \tilde{W}_{c}$ and experimental $\tilde{W}_{c}$ value in Fig. 3(e). Hence within the MQIM-HO the first type of agreement with the ARPES spectra is reached by choosing these parameter values for the electronic density $n_{e}=0.176$.
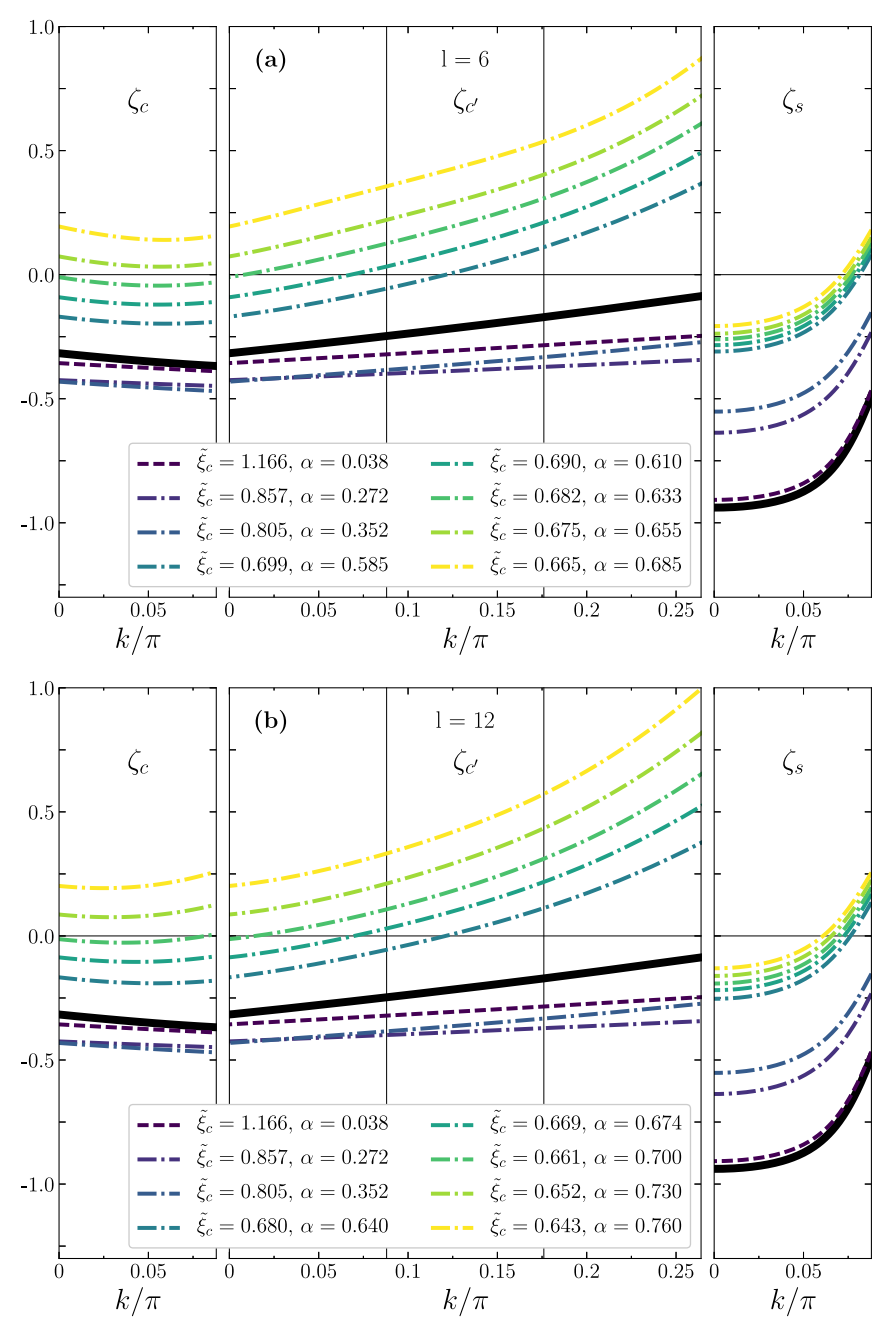

FIG. 4. The exponents, Eq. (A3) of Appendix A, in the spectral function, Eq. (2), that control the line shape near the theoretical $c$, $c^{\prime}$, and $s$ branch lines in Fig. 3(e), respectively, associated with the experimentally observed high-energy $\mathrm{Bi} / \mathrm{InSb}(001)$ ARPES MDC and EDC peaks $[17,18]$. They are here plotted as a function of the momentum $k$ within the MQIM-HO for (a) $l=6$ and (b) $l=12$ at $u=0.30, n_{e}=0.176$, and several $\tilde{\xi}_{c}$ and $\alpha=\left(2-\tilde{\xi}_{c}^{2}\right)^{2} /\left(8 \tilde{\xi}_{c}^{2}\right)$ values. The black solid lines refer to the bare limit, $\tilde{\xi}_{c}=\xi_{c}$ ( $\xi_{c}=$ 1.242 and $\left.\alpha_{0}=0.017\right)$. The black dashed and the dashed-dotted lines correspond to $\alpha<1 / 8$ and $\alpha>1 / 8$ values, respectively. Moreover, $\tilde{\xi}_{c}=0.805,0.857$, and 1.166 refer to $\tilde{\xi}_{c}^{\ominus}=1 / \xi_{c}, \tilde{\xi}_{c}^{\ominus-}$, and $\tilde{\xi}_{c}^{\ominus+}$, respectively. The $\tilde{\xi}_{c}$ values of the lines whose negative exponents ranges agree with the experimentally observed high-energy ARPES $(k, \omega)$-plane MDC and EDC peaks in Fig. 3(e) are those for which the $c^{\prime}$ branch-line exponent crosses zero between $k / \pi=0$ and $k / \pi \approx$ 0.07 . For $l=6$ and 12 , this refers to the small $\tilde{\xi}_{c}$ subintervals $\alpha=0.610-0.633$ and $\alpha=0.674-0.700$, respectively. (Such limiting values are given in Tables IV and V for all $l=6-12$ integers).

\section{Second type of agreement}

The second type of agreement involves the theoretical $\gamma=c, c^{\prime}, s$ exponents $\tilde{\zeta}_{\gamma}(k)$, Eq. (A3) of Appendix A. They are plotted for $u=0.30$ and $n_{e}=0.176$ as a function of the momentum $k$ in Fig. 4(a) for $l=6$ and in Fig. 4(b) for $l=12$. In Appendix $\mathrm{A}$, they are plotted as a function of $k$ for several additional values of $l$. 
The different curves in each figure are associated with different values of the charge parameter $\tilde{\xi}_{c}$ and thus of the SDS exponent $\alpha=\left(2-\tilde{\xi}_{c}^{2}\right)^{2} /\left(8 \tilde{\xi}_{c}^{2}\right)$ and effective range $R_{\text {eff }}$. The black solid lines refer to the bare charge parameter limit, $\tilde{\xi}_{c}=\xi_{c}=1.242$. The values $\tilde{\xi}_{c}=0.805,0.857$, and 1.166 correspond to $\tilde{\xi}_{c}^{\ominus}=1 / \xi_{c}, \tilde{\xi}_{c}^{\ominus-}$, and $\tilde{\xi}_{c}^{\ominus+}$, respectively.

As justified in Sec. V B, we start by finding the $\tilde{\xi}_{c}$ and $l>5$ values at which the second type of agreement is reached. It refers to the momentum intervals (and corresponding energy ranges) at which the exponents $\tilde{\zeta}_{c}(k)$ and $\tilde{\zeta}_{c^{\prime}}(k)$ are negative. Those are required to agree with the corresponding $(k, \omega)$ plane location of the experimentally observed high-energy ARPES MDC and EDC peaks in Figs. 3(e) and 3(f), respectively. This reveals that the integers $l>5$ and the values of the charge parameter $\tilde{\xi}_{c}$ and corresponding SDS exponent $\alpha=$ $\left(2-\tilde{\xi}_{c}^{2}\right)^{2} /\left(8 \tilde{\xi}_{c}^{2}\right)$ for which agreement is reached are those for which the exponents $\tilde{\zeta}_{c}(k)$ and $\tilde{\zeta}_{c^{\prime}}(k)$, Eq. (A3) of Appendix A, in the spectral-function expression near the $c$ and $c^{\prime}$ branch lines, Eq. (2), are negative for $k \in\left[-2 k_{F}+k_{F c}^{\mathrm{ex}}, 2 k_{F}-k_{F c}^{\mathrm{ex}}\right]$ and $k \in\left[-k_{c^{\prime}}^{\text {ex }}, k_{c^{\prime}}^{\text {ex }}\right]$, respectively.

In the case of the exponent $\tilde{\zeta}_{c}(k)$, the momentum $k_{F c}^{\mathrm{ex}}$ appearing in the interval $k \in\left[-2 k_{F}+k_{F c}^{\mathrm{ex}}, 2 k_{F}-k_{F c}^{\mathrm{ex}}\right]$ is such that $k_{F c}^{\text {ex }} / k_{F}$ is vanishing or very small in the thermodynamic limit. It is the experimental value of the small theoretical $c$ band momentum $k_{F c}^{0}=\pi n_{F c}^{0}$ associated with the low density $n_{F c}^{0}$ of $c$ particle scatterers near the $c$ band Fermi points $-2 k_{F}$ and $2 k_{F}$ considered in Sec. III B.

Concerning the momentum interval $k \in\left[-k_{c^{\prime}}^{\text {ex }}, k_{c^{\prime}}^{\text {ex }}\right]$ for which the exponent $\tilde{\zeta}_{c^{\prime}}(k)$ must be negative, there is a small uncertainty in the value of $k_{c^{\prime}}^{\text {ex }}$. It is such that $k_{c^{\prime}}^{\text {ex }} \in\left[0, \delta k_{0}\right]$ where $2 \delta k_{0} \approx 0.10 \AA^{-1}$ in Fig. 3(e) is the momentum width of the ARPES image crossed by the $c^{\prime}$ branch line.

This small uncertainty, which in the units used in the figures corresponds to $\delta k_{0} \in[0,0.07 \pi]$, implies corresponding small uncertainties in the $\tilde{\xi}_{c}$ and $\alpha$ values at which for each $l$ agreement with the experimentally observed high-energy ARPES MDC and EDC peaks is reached. The corresponding two limiting values of such $\tilde{\xi}_{c}$ and $\alpha$ uncertainties at which the exponent $\tilde{\zeta}_{c^{\prime}}(k)$ in Fig. 4 and in Figs. 5 and 6 of Appendix A crosses zero at $k \approx 0$ and $k \approx 0.07 \pi$, respectively, are given in Table IV for each integer $l=6-12$.

Following the direct relation between the $c$ and $c^{\prime}$ branch lines spectra, that $\delta k_{0} \in[0,0.07 \pi]$ ensures that the exponent $\tilde{\zeta}_{c}(k)$ is indeed negative for $k$ intervals $k \in\left[-2 k_{F}+\right.$ $\left.k_{F c}^{\mathrm{ex}}, 2 k_{F}-k_{F c}^{\mathrm{ex}}\right]$ where $k_{F c}^{\mathrm{ex}} / k_{F} \ll 1$, as also required for the second type of agreement to be reached.

Hence regarding the $c$ and $c^{\prime}$ branch lines, agreement between theory and experiments is reached by the $\tilde{\xi}_{c}$ and $l>5$ values that in Fig. 4 and in Figs. 5 and 6 of Appendix A correspond to the $c^{\prime}$ branch line exponent curves crossing zero between $k \approx 0$ and $k \approx \delta k_{0} \approx 0.07 \pi$. (In such figures, only the two corresponding $c^{\prime}$ branch line exponent curves crossing zero at $k \approx 0$ and $k \approx \delta k_{0} \approx 0.07 \pi$, respectively, are plotted).

The theoretical $s$ branch line exponent $\tilde{\zeta}_{s}(k)$, Eq. (A3) of Appendix A, does not depend on the integer quantum number $l>5$. For the $\tilde{\xi}_{c}$ values for which the $c^{\prime}$ branch line exponent curves cross zero between $k \approx 0$ and $k \approx \delta k_{0} \approx 0.07 \pi$ in Fig. 4 and in Figs. 5 and 6 of Appendix A, the exponent $\tilde{\zeta}_{s}(k)$ is negative in corresponding intervals $k \in]-k_{F}+k_{F s}^{*}, k_{F}-$ $k_{F s}^{*}[$ and thus positive for $\left.|k| \in]\left(k_{F}-k_{F s}^{*}\right), k_{F}\right]$. Here, $k_{F s}^{*}$ is
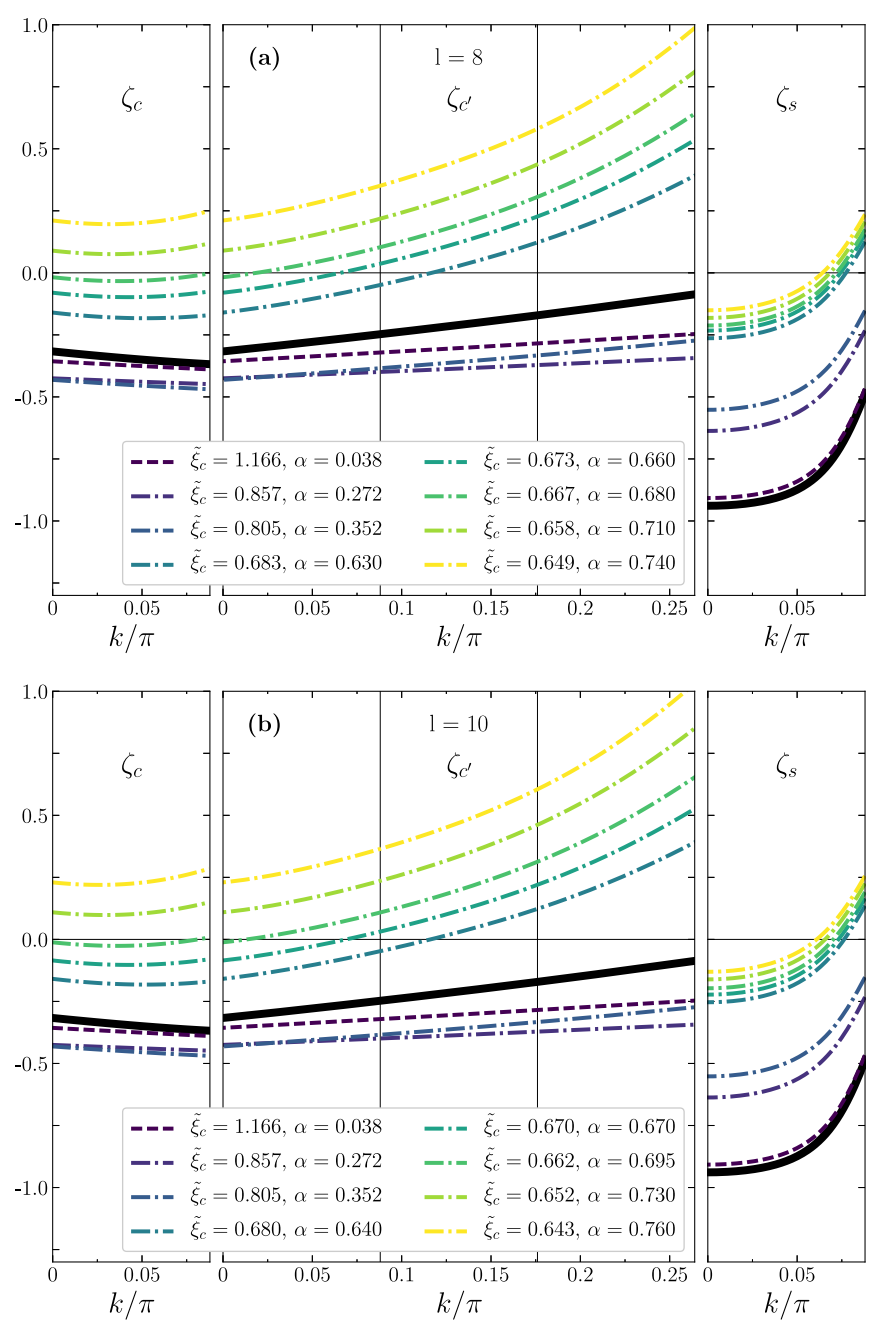

FIG. 5. The same exponents as in Fig. 4 for (a) $l=8$ and (b) 10 . The choice of the $\tilde{\xi}_{c}$ intervals corresponding to the lines whose negative exponents ranges agree with the experimentally observed high-energy ARPES $(k, \omega)$-plane MDC and EDC peaks in Fig. 3(e) obeys the same criterion as in that figure. For $l=8$ and 10 , such intervals whose limiting values are given in Tables IV and $\mathrm{V}$ are $\alpha=0.660-0.680$ and $0.670-0.695$, respectively.

a function of $n_{e}, u$, and $\tilde{\xi}_{c}$ and $k= \pm\left(k_{F}-k_{F s}^{*}\right)$ are the two momentum values at which $\tilde{\zeta}_{s}(k)$ vanishes.

The predicted location at $k \in]-k_{F}+k_{F s}^{*}, k_{F}-k_{F s}^{*}[$ of the ARPES MDC peaks associated with the $s$ branch line cannot be confirmed from the available experimental data. Indeed and as mentioned in Sec. V B, it is not possible to extract from such data the dispersion of that line. However, the corresponding energy intervals $|\omega| \in\left[\left|\tilde{\omega}_{s}\left(k_{F}-k_{F s}^{*}\right)\right|, \tilde{W}_{s}\right]$ are consistent with the available experimental data from the EDCs in Fig. 3(d). Here $|\omega|=\tilde{W}_{s}=\left|\tilde{\omega}_{s}(0)\right|$ is the bottom of the $s$ branch line energy, as estimated in Sec. V C 1 from the interplay of the kinematical constraints, Eq. (30), and the ARPES MDC shown in Fig. 3(c) for $|\omega|=0.05 \mathrm{eV}$.

\section{Third type of agreement}

From the above results we see that for $l=6-12$ agreement with the experimentally observed high-energy ARPES MDC 

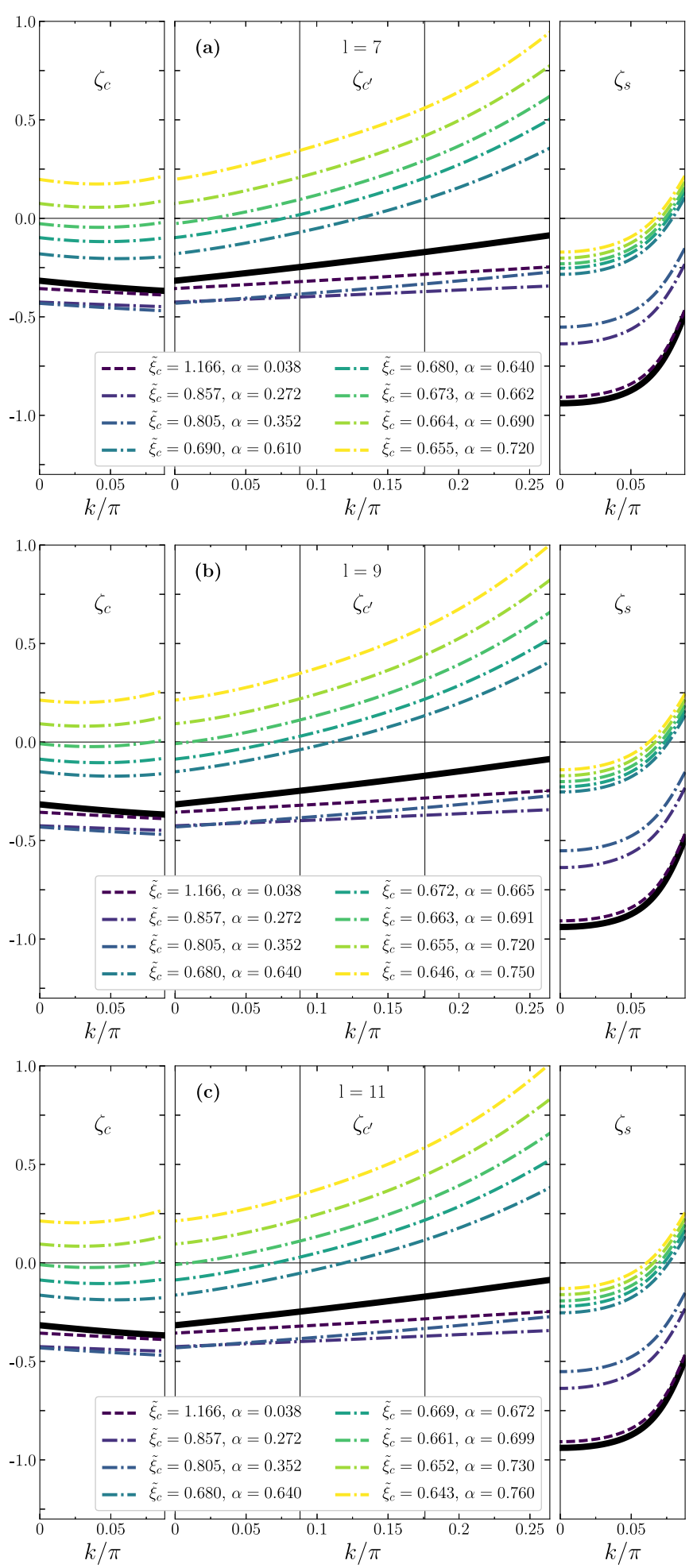

FIG. 6. The same exponents as in Figs. 4 and 5 for (a) $l=7$, (b) 9 , and (c) 11 . The choice of the $\tilde{\xi}_{c}$ intervals corresponding to the lines whose negative exponents ranges agree with the experimentally observed high-energy ARPES $(k, \omega)$-plane MDC and EDC peaks in Fig. 3(e) obeys the same criterion as in Fig. 4. For $l=7,9$, and 11 such intervals whose limiting values are given in Tables IV and $\mathrm{V}$ are $\alpha=0.640-0.662,0.665-0.691$, and 0.672-0.699, respectively.

and EDC peaks in Figs. 3(e) and 3(f) is reached by the exponents curves referring to $\tilde{\xi}_{c}$ and $\alpha$ values belonging to
TABLE IV. The two values of the charge parameter $\tilde{\xi}_{c}$ and corresponding SDS exponent $\alpha=\left(2-\tilde{\xi}_{c}^{2}\right)^{2} /\left(8 \tilde{\xi}_{c}^{2}\right)$ that at each integer $l=6-12$ are those at which the exponent $\tilde{\zeta}_{c^{\prime}}(k)$ plotted as a function of $k$ in Fig. 4(a) for $l=6$ and in Fig. 4(b) for $l=12$ crosses zero at $k \approx 0$ and $\approx 0.07 \pi$, respectively. The same applies to the exponent $\tilde{\zeta}_{c^{\prime}}(k)$ plotted for $l=7-11$ in Figs. 5(a), 5(b), and 6(a)-6(c) of Appendix A.

\begin{tabular}{lcccc}
\hline \hline$l$ & $\tilde{\xi}_{c}$ & $\alpha$ & $\tilde{\xi}_{c}$ & $\alpha$ \\
\hline & $\left(\tilde{\zeta}_{c^{\prime}}(0)=0\right)$ & $\left(\tilde{\zeta}_{c^{\prime}}(0)=0\right)$ & $\left(\tilde{\zeta}_{c^{\prime}}\left(\frac{7 \pi}{100}\right)=0\right)$ & $\left(\tilde{\zeta}_{c^{\prime}}\left(\frac{7 \pi}{100}\right)=0\right)$ \\
6 & 0.682 & 0.633 & 0.690 & 0.610 \\
7 & 0.673 & 0.662 & 0.680 & 0.640 \\
8 & 0.667 & 0.680 & 0.673 & 0.660 \\
9 & 0.663 & 0.691 & 0.672 & 0.665 \\
10 & 0.662 & 0.695 & 0.67 & 0.670 \\
11 & 0.661 & 0.699 & 0.669 & 0.672 \\
12 & 0.661 & 0.700 & 0.669 & 0.674 \\
\hline \hline
\end{tabular}

the small intervals reported in TableV. The overlap of the subintervals obtained for each $l=6-12$ given in that table then leads to the theoretical predictions $\tilde{\xi}_{c} \in[0.66,0.69]$ and $\alpha \in$ [0.610-0.700].

Table $\mathrm{V}$ also provides the corresponding intervals of the effective range $R_{\text {eff }}$ in units of the lattice spacing that refer to first and second types of agreements. The effective range dependence on the bare charge parameter $\xi_{c}=\xi_{c}\left(n_{e}, u\right)$, renormalized charge parameter $\tilde{\xi}_{c}$, and integer quantum number $l>5$ values at which such agreements have been reached is defined by combining Eqs. (10) and (26). That table also provides the values of the length scale $2 r_{l}$ in the same units whose dependence on $l$ is given in Eq. (29). Upon increasing $l$ from $l=6$ to $l=12$, the effective range $R_{c}^{\text {eff }}$ values for which there is agreement with the experiments change from $R_{c}^{\text {eff }} \approx 5 r_{l}$ to $R_{c}^{\text {eff }} \approx r_{l}$, respectively.

TABLE V. The renormalized charge parameter $\tilde{\xi}_{c}$, SDS exponent $\alpha$, and effective range $R_{c}^{\text {eff }}$ intervals for which there is agreement between the $(k, \omega)$-plane regions where the theoretical branch lines display singularities and the corresponding experimentally observed high-energy $\mathrm{Bi} / \mathrm{InSb}(001)$ ARPES MDC and EDC peaks in Figs. 3(e) and 3(f) for $n_{e}=0.176, u=0.30$, and $l=6-12$. As given in Table IV, for each integer $l$ the smallest and largest $\tilde{\xi}_{c}$ value refers to the largest and smallest corresponding $\alpha=\left(2-\tilde{\xi}_{c}^{2}\right)^{2} /\left(8 \tilde{\xi}_{c}^{2}\right)$ value, respectively. (The $\alpha$ values were derived using more digits in the $\tilde{\xi}_{c}$ values than given in the table). The values in units of $a_{0}=1$ of the length scale $2 r_{l}$, Eq. (29), and related parameter $\sqrt{2 \mu \gamma_{c}}=$ $\sqrt{2}\left(2 r_{l}\right)^{\frac{l-2}{2}}$ are also provided.

\begin{tabular}{lccccc}
\hline \hline$l$ & $\tilde{\xi}_{c}$ & $\alpha$ & $R_{\text {eff }} / a_{0}$ & $2 r_{l} / a_{0}$ & $\sqrt{2 \mu \gamma_{c}}$ \\
\hline 6 & $0.68-0.69$ & $0.610-0.633$ & $14.4-17.0$ & 6.0 & 50.1 \\
7 & $0.67-0.68$ & $0.640-0.662$ & $6.9-8.1$ & 6.3 & 140.5 \\
8 & 0.67 & $0.660-0.680$ & $5.0-5.8$ & 6.4 & 377.2 \\
9 & $0.66-0.67$ & $0.665-0.691$ & $4.0-4.8$ & 6.5 & 983.3 \\
10 & $0.66-0.67$ & $0.670-0.695$ & $3.4-4.2$ & 6.5 & $2.51 \times 10^{3}$ \\
11 & $0.66-0.67$ & $0.672-0.699$ & $3.1-3.8$ & 6.5 & $6.29 \times 10^{3}$ \\
12 & $0.66-0.67$ & $0.674-0.700$ & $2.9-3.5$ & 6.4 & $1.56 \times 10^{4}$ \\
\hline \hline
\end{tabular}


According to the analysis of Sec. V C 2, agreement with the experimentally observed high-energy $(k, \omega)$-plane ARPES MDC and EDC peaks distribution has been reached for the SDS exponent range $\alpha \in[0.610-0.700]$. The third type of agreement between theory and experiments defined in Sec. V C 2 is reached provided that such a predicted SDS exponent range agrees with the $\alpha$ values measured within the low-energy angle integrated photoemission intensity. An experimental uncertainty $\alpha=0.65 \pm 0.05$ of the SDS exponent was found for $-\omega<0.1 \mathrm{eV}$ in Ref. [17].

The remarkable quantitative agreement of the MQIM-HO predictions within the third criterion reported in Sec. V C 2 provides evidence of finite-range interactions playing an active role in the $\mathrm{Bi} / \mathrm{InSb}(001)$ spectral properties and confirms the 1D character of its metallic states also found in Ref. [17].

\section{Interplay of relaxation processes with the momentum dependence of the exponents}

Here we discuss the physical mechanisms within the MQIM-HO that underlie the dependence of the exponents $\tilde{\zeta}_{c}(k), \tilde{\zeta}_{c^{\prime}}(k)$, and $\tilde{\zeta}_{s}(k)$ on the charge parameter $\tilde{\xi}_{c}$. These exponents are plotted in Fig. 4 and in Figs. 5 and 6 of Appendix A.

In the bare charge parameter limit, $\tilde{\xi}_{c}=\xi_{c}$, the exponents being negative or positive just refers to a different type of power-law behavior near the corresponding charge and spin branch lines. For $\tilde{\xi}_{c}<\xi_{c}$, this applies only to the spin $s$ branch line. It coincides with the edge of support of the one-electron removal spectral function that separates $(k, \omega)$-plane regions without and with finite spectral weight. Hence conservation laws impose that, near that line, the spectral function remains of power-law form, Eq. (2), for both intervals $\left.\tilde{\xi}_{c} \in\right] 1 / 2,1[$ and $\left.\left.\tilde{\xi}_{c} \in\right] 1, \xi_{c}\right]$. As confirmed from an analysis of the $s$ branch-line exponents plotted in Fig. 4 and in Figs. 5 and 6 of Appendix A, the effect of decreasing the charge parameter $\tilde{\xi}_{c}$ from its initial bare value $\xi_{c}$ (and thus increasing the SDS exponent $\alpha=(2-$ $\left.\tilde{\xi}_{c}^{2}\right)^{2} /\left(8 \tilde{\xi}_{c}^{2}\right)$ from $\left.\alpha_{0}=\left(2-\xi_{c}^{2}\right) /\left(8 \xi_{c}^{2}\right) \in[0,1 / 8]\right)$ is merely to increase the spin branch line exponent $\tilde{\zeta}_{s}(k)$. Except for two regions near $-k_{F}$ and $k_{F}$ corresponding to $|k| \in\left[\left(k_{F}-\right.\right.$ $\left.\left.k_{F s}^{*}\right), k_{F}\right]$, that exponent remains negative, so that the singularities prevail. In the complementarily small momentum regions near $\pm k_{F}$ defined by $|k| \in\left[\left(k_{F}-k_{F s}^{*}\right), k_{F}\right]$ where the exponent is positive, the line shape remains of power-law type.

Analysis of the $c$ and $c^{\prime}$ branch-line exponents curves plotted in the same figures reveals that the situation is different for the one-electron removal spectral function in the vicinity of the charge $c$ and $c^{\prime}$ branch lines, Eq. (2). These are located in the continuum of the one-electron spectral function. The physics is though different for the subintervals $\left.\left.\tilde{\xi}_{c} \in\right] 1, \xi_{c}\right]$ and $\left.\tilde{\xi}_{c} \in\right] 1 / 2,1$ [, respectively.

Smoothly decreasing $\tilde{\xi}_{c}$ from its initial bare value $\xi_{c}$ to $\tilde{\xi}_{c} \rightarrow 1$, produces effects quite similar to those of increasing $U$ within the 1D Hubbard model to $U \rightarrow \infty$ [12]. Indeed, these changes render $\tilde{\zeta}_{c}(k)$ and $\tilde{\zeta}_{c^{\prime}}(k)$ more negative and lead to an increase of the width of the $k$ intervals in which they are negative. Within the $\left.\tilde{\xi}_{c} \in\right] 1, \xi_{c}$ ] interval, a large number of $\tilde{\xi}_{c}=\xi_{c}$ conservation laws that are behind the factorization of the scattering $S$ matrix into two-particle scattering processes survive, which tend to prevent the $c$ impurity from undergoing relaxation processes. Hence the lifetimes $\tau_{c}(k)$ and $\tau_{c^{\prime}}(k)$ in Eq. (2) are very large for the $k$ intervals for which the corresponding branch line exponents are negative, so that the expression given in the equation for the spectral function near the $\beta=c, c^{\prime}$ branch lines is nearly power-law-like, $\tilde{B}(k, \omega) \propto$ $\left(\tilde{\omega}_{\beta}(k)-\omega\right)^{\tilde{\zeta}_{\beta}(k)}$.

The effects of the finite-range interactions increase upon decreasing $\tilde{\xi}_{c}$ within the interval $\tilde{\xi}_{c} \in\left[\tilde{\xi}_{c}^{\ominus}, 1\left[\right.\right.$ where $\tilde{\xi}_{c}^{\oslash}=$ $1 / \xi_{c}=0.805$ for $n_{e}=0.176$ and $u=0.30$. Indeed, smoothly decreasing $\tilde{\xi}_{c}$ within that interval tends to remove an increasing number of conservation laws, which strengthens the effects of the impurity relaxation processes. Such effects become more pronounced when $\Delta a / \tilde{a} \in]-1,0[$ and $\tan (\Phi)>0$, upon further decreasing $\tilde{\xi}_{c}$ within the interval $\left.\left.\tilde{\xi}_{c} \in\right] 1 / 2, \tilde{\xi}_{c}^{\oslash}\right]$.

In the $k$ intervals for which the $\beta=c, c^{\prime}$ branch line exponents $\tilde{\zeta}_{\beta}(k)$ remain negative, the lifetimes $\tau_{\beta}(k)$ in Eq. (2) remain large and the $c$ impurity relaxation processes only slightly broaden the spectral-function power-law singularities, as given in Eq. (2). For the complementary $k$ ranges for which such exponents become positive upon decreasing $\tilde{\xi}_{c}$ and thus increasing $\alpha$, the high-energy singularities are rather washed out by the relaxation processes.

As confirmed by analysis of the curves plotted in Fig. 4 and in Figs. 5 and 6 of Appendix A, starting at $|k|=3 k_{F}-$ $k_{F c}^{0}$ and downwards, smoothly decreasing $\widetilde{\xi}_{c}$ from $\tilde{\xi}_{c}^{\ominus}$ first gradually enhances the $k$ domains where $\tilde{\zeta}_{c^{\prime}}(k)$ is positive. Further decreasing $\tilde{\xi}_{c}$ after the $c^{\prime}$ branch line singularities are fully washed out leads to the emergence of a $c$ branch line $k$ domain starting at $|k|=0$ and upwards in which that line singularities are finally fully washed out up to $|k|=k_{F}-k_{F c}^{0}$ below a smaller $\tilde{\xi}_{c}$ value.

\section{DISCUSSION AND CONCLUDING REMARKS}

\section{A. Discussion of other effects and properties outside the range of the MQIM-HO}

As reported in Sec. V A, the ARPES data were taken at $8 \mathrm{~K}$ and the angle integrations to detect the suppression of the photoelectron intensity were performed at $k_{y}=0.2 \AA^{-1}$, near the boundary of the $(1 \times 3)$ surface Brillouin zone $\left(0.23 \AA^{-1}\right)$.

As shown in Fig. 2(b) of Ref. [17], at $k_{y}=0.2 \AA^{-1}$, there is an energy gap between the spectral features studied in this paper within a 1D theoretical framework and a bulk valence band. Due to that energy gap, the coupling between the two problems is negligible, which justifies that the system studied here corresponds to $1 \mathrm{D}$ physics.

Smoothly changing $k_{y}$ from $k_{y}=0.2 \AA^{-1}$ to $k_{y}=0$ corresponds to smoothly turning on the coupling to the $2 \mathrm{D}$ physics. As shown in Fig. 2(a) of Ref. [17], at $k_{y}=0$ the energy gap between the spectral features studied in this paper and that bulk valence band has been closed. The study of the microscopic mechanisms involved in the physics associated with turning on the coupling to the $2 \mathrm{D}$ physics by smoothly changing $k_{y}$ from $k_{y}=0.2 \AA^{-1}$ to $k_{y}=0$ is an interesting problem that deserves further investigation. 
Another interesting open problem refers to theoretical prediction of the MDC for extended momentum intervals and of the EDC for corresponding energy ranges. The universal form of the spectral function near the singularities, Eq. (2), is determined by the large $x$ behavior of the potential $V_{c}(x)$, Eq. (5), which follows from that of the potential $V_{e}(r)$ in Eq. (1), and potential sum rules, Eqs. (6) and (7). As reported in Eqs. (12) and (13), the value of the renormalized charge parameter $\tilde{\xi}_{c}$ behind the renormalization of the phase shifts in the exponents of that spectral function expression, Eq. (2), is indeed controlled by the value of the initial bare charge parameter $\xi_{c}=\xi_{c}\left(n_{e}, u\right)$, the integer quantum number $l>5$ associated with the potential $V_{c}(x)$ large- $x$ behavior, Eq. (5), and the zero-energy phase $\Phi$ determined by that potential sum rules, Eqs. (6) and (7). Plotting a MDC for extended momentum intervals and an EDC for corresponding energy ranges is a problem that involves nonuniversal properties of the one-electron removal spectral function. This would require additional information of that function in $(k, \omega)$-plane regions where it is determined by the detailed nonuniversal dependence on $r$ of the specific electronic potential $V_{e}(r)$ suitable to $\mathrm{Bi} / \mathrm{InSb}(001)$.

Another interesting issue refers to the validity of the MQIM-HO to describe the $\mathrm{Bi} / \mathrm{InSb}(001)$ one-electron spectral properties. The question is whether the interplay of one dimensionality and electron finite-range interactions is indeed the main microscopic mechanism behind such properties. As in all lattice electronic condensed matter systems, it is to be expected that there are both some degree of disorder effects and electron-electron effects in the $\mathrm{Bi} / \mathrm{InSb}(001)$ physics. However, we can provide evidence that the interplay of latter effects with the $\mathrm{Bi} / \mathrm{InSb}(001)$ metallic states one dimensionality is the dominant contribution to the one-electron removal spectral properties.

The first strong evidence that this is so is the experimentally observed universal power-law scaling of the spectral intensity $I(\omega, T)$. (Here, $\omega=0$ refers to the Fermi-level energy). For instance, at $\omega=0$ and finite $T$ and at $T=0$ and low $\omega$, it was found in Ref. [17] to have the following TLL behaviors for $\mathrm{Bi} / \mathrm{InSb}(001)$,

$$
I(0, T) \propto T^{\alpha} \text { and } I(\omega, 0) \propto|\omega|^{\alpha},
$$

respectively, where $\alpha$ is the SDS exponent.

If there were important effects from disorder, its interplay with electron-electron interactions would rather give rise in the case of $1 \mathrm{D}$ and quasi-1D systems to a spectral intensity $I(\omega, T)$ with the following behaviors [34-36]:

$$
\begin{aligned}
& I(0, T) \propto e^{-\sqrt{\frac{C_{0}^{2}}{16 \pi D_{0} T}} \text { and }} \\
& I(\omega, 0) \propto|\omega|^{1 / 2} \frac{\sqrt{32 \pi D_{0}}}{C_{0}} e^{-\frac{C_{0}^{2}}{32 \pi D_{0}|\omega|}},
\end{aligned}
$$

for $\omega \ll C_{0}^{2} /\left(32 \pi D_{0}\right)$. Here, $D_{0} \propto l$ is the bare diffusion coefficient and $C_{0}$ is a constant that depends on the effective electron-electron interaction and electronic density.

The behaviors in Eq. (32) are qualitatively different from those reported in Eq. (31), which are those experimentally observed in $\mathrm{Bi} / \mathrm{InSb}(001)$. This holds specially for $I(0, T)$, in which case disorder effects cannot generate such a temperature power-law scaling. Also the experimentally found behavior $I(\omega, 0) \propto|\omega|^{\alpha}$ disagrees with that implied by Eq. (32).

Further, in the limit of low $\omega$ and $T$, the MQIM-HO describes the corresponding TLL limit in which the universal power-law scaling of the spectral intensity $I(\omega, T)$ has the behaviors reported in Eq. (31). Theoretically, the value of the SDS exponent $\alpha$ depends on those of the electronic density $n_{e}$, the interaction $u=U / 4 t$, and the renormalized charge parameter $\tilde{\xi}_{c}$. Within the MQIM-HO phase shifts constraints, its values span the intervals $\alpha \in\left[\alpha_{0}, 1 / 8[;] 1 / 8,49 / 32[\right.$.

The theoretically predicted $\alpha$ value has been determined from agreement of the $T=0$ one-electron spectral function, Eq. (2), with the ARPES peaks location in the $(k, \omega)$ plane. The quantitative agreement then reached refers to the experimental value $\alpha=0.65 \pm 0.05$ obtained for $I(\omega, 0) \propto|\omega|^{\alpha}$ at $|\omega|<0.1 \mathrm{eV}$ in Ref. [17]. For low temperatures and $\omega=0$, the MQIM-HO also leads to the $I(0, T)$ behavior given in Eq. (31).

Finally, despite bismuth $\mathrm{Bi}$, indium In, and antimony $\mathrm{Sb}$ being heavy elements, the present 1D surface metallic states do not show any detectable spin-orbit coupling effects and nor any related Rashba-split bands. In this regard, it is very important to distinguish the system studied in this paper with 1-2 monatomic layers of $\mathrm{Bi}$ thickness whose ARPES data were first reported in Ref. [17] from the system with a similar chemical name which was studied in Ref. [37], which refers to 5-20 monatomic layers of Bi thickness. One expects, and indeed observes, significant qualitative differences in these two systems.

\section{B. Concluding remarks}

In this paper, we have discussed an extension of the MQIM-LO used in the theoretical studies of the ARPES in the line defects of $\mathrm{MoSe}_{2}$ [16]. This MQIM-type approach [6,7] accounts only for the renormalization of the leading term in the effective range expansion of the charge-charge phase shift, Eq. (4). As shown in Ref. [24], this is a good approximation if the effective range of the interactions of the $c$ particles and the $c$ impurity is of about one lattice spacing.

The MQIM-HO developed in this paper accounts for the renormalization of the higher terms in the effective range expansion of the charge-charge phase shift, Eq. (4). It applies to a class of 1D lattice electronic systems described by the Hamiltonian, Eq. (1), which has longer range interactions. The quantum problem described by that Hamiltonian is very involved in terms of many-electron interactions. However, we found that a key simplification is the unitary limit associated with the scattering of the fractionalized charged particles by the $c$ impurity. We have shown a theory based on the MQIM-HO with finite-range interactions, Eq. (1), applies to the study of some of the one-electron spectral properties of $\mathrm{Bi} / \mathrm{InSb}(001)$ measured at $y$ momentum component $k_{y}=$ $0.2 \AA^{-1}$ and temperature $8 \mathrm{~K}$.

Consistent with the relation of the electron and $c$ particle representations discussed in Appendix $\mathrm{C}$, the form of the attractive potential $V_{c}(x)$ associated with the interaction of the $c$ particles and the $c$ impurity at a distance $x$ is determined by that of the electronic potential $V_{e}(r)$ in Eq. (1). The universal behavior of the spectral function near the singularities given in Eq. (2) whose $(k, \omega)$-plane location refers to that of the 
experimentally observed high-energy ARPES peaks, is determined by the large $x$ behavior of $V_{c}(x)$ shown in Eq. (5) and sum rules, Eqs. (6) and (7). Otherwise the spectral function expression in the continuum is not universal.

Despite the limited available experimental information about the ARPES peaks located on the spin branch line, we have shown that all the three criteria associated with the different types of agreement between theory and experiments considered in Sec. V B are satisfied. This provides further evidence to that given in Ref. [17] for the interplay of one dimensionality and finite-range interactions playing an important role in the one-electron spectral properties of the metallic states in $\mathrm{Bi} / \mathrm{InSb}(001)$.

\section{ACKNOWLEDGMENTS}

J.M.P.C. acknowledges the late Adilet Imambekov for discussions that were helpful in producing the theoretical part of the paper. Y.O. and S.-i.K. thank illuminating discussions to obtain and analyze the ARPES dataset by Patrick Le Fèvre, François Bertran, and Amina Taleb-Ibrahimi. J.M.P.C. would like to thank Boston University's Condensed Matter Theory Visitors Program for support and the hospitality of MIT. J.M.P.C. and T.C. acknowledge the support from Fundação para a Ciência e Tecnologia (FCT) through the Grants UID/FIS/04650/2013, PTDC/FIS-MAC/29291/2017, and SFRH/BSAB/142925/2018, NSAF U1530401 and computational resources from Computational Science Research Center (CSRC) (Beijing), and the National Science Foundation of China (NSFC) Grant 11650110443.

\section{APPENDIX A: USEFUL QUANTITIES}

The spectra of the $\gamma=s, c, c^{\prime}$ branch lines in the expressions for the one-electron removal spectral function in Eq. (2) have for the MQIM-HO the same general form as for the MQIM-LO [16,24] and read

$$
\begin{aligned}
\tilde{\omega}_{s}(k) & =\tilde{\varepsilon}_{s}(k)=\varepsilon_{s}(k) \leqslant 0 \text { for } k=-q^{\prime} \in\left[-k_{F}, k_{F}\right], \\
\tilde{\omega}_{c}(k) & =\tilde{\varepsilon}_{c}\left(|k|+k_{F}\right) \leqslant 0 \text { for } \\
k & =k_{c}=-\operatorname{sgn}\{k\} k_{F}-q \in\left[-k_{F}, k_{F}\right], \\
\tilde{\omega}_{c^{\prime}}(k) & =\tilde{\varepsilon}_{c}\left(|k|-k_{F}\right) \leqslant 0 \text { for } \\
k & =k_{c^{\prime}}=\operatorname{sgn}\{k\} k_{F}-q \in\left[-3 k_{F}, 3 k_{F}\right] .
\end{aligned}
$$

Here, $\tilde{\varepsilon}_{s}\left(q^{\prime}\right)$ and $\tilde{\varepsilon}_{c}(q)$ are the $s$ and $c$ particle energy dispersions, respectively. For the $c$ and $s$ band momentum intervals at which the $c$ and $s$ impurities, respectively, are created under one-electron excitations the energy dispersions and corresponding group velocities read

$$
\begin{aligned}
& \tilde{\varepsilon}_{c}(q)=\left(1+\beta_{c}\right) \varepsilon_{c}(q) \text { for } q \in\left[-2 k_{F}, 2 k_{F}\right], \\
& \tilde{\varepsilon}_{s}\left(q^{\prime}\right)=\varepsilon_{s}\left(q^{\prime}\right) \text { for } q^{\prime} \in\left[-k_{F}, k_{F}\right], \\
& \tilde{v}_{\beta}(q)=\frac{\partial \tilde{\varepsilon}_{\beta}(q)}{\partial q}, \quad v_{\beta}(q)=\frac{\partial \varepsilon_{\beta}(q)}{\partial q}, \quad \beta=c, s .
\end{aligned}
$$

Here, the bare $\tilde{\xi}_{c}=\xi_{c}$ energy dispersions $\varepsilon_{c}(q)$ and $\varepsilon_{s}\left(q^{\prime}\right)$ are defined below and $\beta_{c}=\sqrt{1+\alpha_{c}}-1$, where $\alpha_{c}$ is given in Eq. (C6) of Appendix C. It reads $\beta_{c}=\left(\xi_{c}^{2}-\tilde{\xi}_{c}^{2}\right) / \tilde{\xi}_{c}^{2}$ for $\tilde{\xi}_{c}>\tilde{\xi}_{c}$ whereas $\beta_{c}=\left(2-\xi_{c}^{2}\right) / \xi_{c}^{2}$ for the range $\tilde{\xi}_{c}<\tilde{\xi}_{c}$ of most interest for our studies where $\tilde{\xi}_{c}=\xi_{c}^{2} / \sqrt{2}$. For the latter range, its limiting behaviors are

$$
\begin{aligned}
\beta_{c} & =\frac{U}{4 \pi t \sin k_{F}} \text { for } u \ll 1 \\
& =1-\frac{8 \ln 2}{\pi U} 2 t \sin \left(2 k_{F}\right) \text { for } u \gg 1 .
\end{aligned}
$$

The renormalization $\tilde{\varepsilon}_{c}(q)=\left(1+\beta_{c}\right) \varepsilon_{c}(q)$ in Eq. (A2) is related to the expression $\lim _{k \rightarrow 0} \mathcal{V}_{c}(k)=\frac{\pi}{2} \alpha_{c} v_{c}\left(2 k_{F}\right)$, Eq. (C6) of Appendix $\mathrm{C}$, and the corresponding ratio $\tilde{v}_{c}\left(2 k_{F}\right) / v_{c}\left(2 k_{F}\right)=\sqrt{1+\alpha_{c}}=\left(1+\beta_{c}\right)$ of the renormalized and bare $c$ band Fermi velocities [38]. That the spin dispersion $\tilde{\varepsilon}_{s}\left(q^{\prime}\right)$ remains invariant under finite-range interactions whereas the charge dispersion bandwidth $\tilde{W}_{c}^{p}=-\tilde{\varepsilon}_{c}(0)$ and the charge Fermi velocity $\tilde{v}_{c}\left(2 k_{F}\right)$ are slightly increased as the range of interactions increases is known from numerical studies [39]. (See the related charge and spin spectra in Fig. 7 of Ref. [39] and the corresponding discussion).

In the MQIM-HO, the momentum dependent $\gamma=c, c^{\prime}, s$ exponents $\tilde{\zeta}_{\gamma}(k)$ in the expressions for the one-electron removal spectral function, Eq. (2), also have the same form as for the MQIM-LO. However, some of the quantities in their following expressions have additional MQIM-HO terms:

$$
\begin{aligned}
\tilde{\zeta}_{c}(k) & =-\frac{1}{2}+\sum_{\iota= \pm 1}\left(\frac{\xi_{c}}{4}-\tilde{\Phi}_{c, c}\left(\imath 2 k_{F}, q\right)\right)^{2}, \text { where } \\
k & =\operatorname{sgn}\{q\} k_{F}-q \in\left[-k_{F}+k_{F c}^{0}, k_{F}-k_{F c}^{0}\right] \\
q & =-\operatorname{sgn}\{k\} k_{F}-k \in\left[-2 k_{F}+k_{F c}^{0},-k_{F}\right] \text { and } \\
& =-\operatorname{sgn}\{k\} k_{F}-k \in\left[k_{F}, 2 k_{F}-k_{F c}^{0}\right] ; \\
\tilde{\zeta}_{c^{\prime}}(k) & =-\frac{1}{2}+\sum_{\iota= \pm 1}\left(\frac{\tilde{\xi}_{c}}{4}-\tilde{\Phi}_{c, c}\left(\imath 2 k_{F}, q\right)\right)^{2}, \text { where } \\
k & =-\operatorname{sgn}\{q\} k_{F}-q \in\left[-3 k_{F}+k_{F c}^{0}, 3 k_{F}-k_{F c}^{0}\right] \\
q & =\operatorname{sgn}\{k\} k_{F}-k \in\left[-2 k_{F}+k_{F c}^{0}, k_{F}\right] \text { and } \\
& =\operatorname{sgn}\{k\} k_{F}-k \in\left[-k_{F}, 2 k_{F}-k_{F c}^{0}\right] ; \\
\tilde{\zeta}_{s}(k) & =-1+\sum_{\iota= \pm 1}\left(-\frac{\iota}{2 \tilde{\xi}_{c}}-\tilde{\Phi}_{c, s}\left(\iota 2 k_{F}, q^{\prime}\right)\right)^{2}, \quad \text { where } \\
k & =-q^{\prime} \in\left[-k_{F}+k_{F s}^{0}, k_{F}-k_{F s}^{0}\right], \\
q^{\prime} & =-k \in\left[-k_{F}+k_{F s}^{0}, k_{F}-k_{F s}^{0}\right] .
\end{aligned}
$$

These exponents are plotted in Figs. 4(a) and 4(b) as a function of the momentum $k$ within the MQIM-HO for $u=0.30$, $n_{e}=0.176$, and even values $l=6$ and 12 , respectively. In this Appendix they are plotted in Figs. 5 and 6 for even values $l=8,10$ and odd values $l=7,9,11$, respectively.

The phase shifts $2 \pi \tilde{\Phi}_{c, s}\left( \pm 2 k_{F}, q^{\prime}\right)$ and $2 \pi \tilde{\Phi}_{c, c}\left( \pm 2 k_{F}, q\right)$ that in Eq. (A3) appear in units of $2 \pi$ are defined in Eqs. (3) and (14), respectively. The bare phase shifts $2 \pi \Phi_{c, s}\left( \pm 2 k_{F}, q^{\prime}\right)$ and $2 \pi \Phi_{c, c}\left( \pm 2 k_{F}, q\right)$ in the latter equations are defined below. The MQIM-HO phase shift term $2 \pi \tilde{\Phi}_{c, c}^{R_{\text {eff }}}\left(k_{r}\right)$ in Eq. (14) accounts for effects of finite-range interactions beyond the MQIM-LO through the spectral function exponents $\tilde{\zeta}_{c}(k)$ and $\tilde{\zeta}_{c^{\prime}}(k)$ in Eq. (A3).

The small momentum $k_{F s}^{0}$ in Eq. (A3) such that $k_{F s}^{0} / k_{F} \ll$ 1 is in general smaller than the momentum $k_{F s}^{*}$ considered in 
the discussions of Sec. V C 2. It has the same role for the $s$ band as $k_{F c}^{0}$ for the $c$ band, concerning the crossover between the low-energy TLL and high-energy regimes. As discussed below in Appendix B, for physical momenta $k$ associated with creation of the $c$ impurity at $q$ in the small $c$ band momentum in the absolute value interval $|q| \in\left[2 k_{F}-k_{F c}^{0}, 2 k_{F}\right]$ and creation of the $s$ impurity at $q^{\prime}$ in the small $s$ band momentum absolute value interval $\left|q^{\prime}\right| \in\left[k_{F}-k_{F s}^{0}, k_{F}\right]$ corresponding to the low-energy TLL regime the expressions for the exponents, Eq. (A3), are not valid.

The bare energy dispersions $\varepsilon_{c}(q)$ and $\varepsilon_{s}\left(q^{\prime}\right)$ in Eq. (A2) are defined as follows:

$$
\begin{aligned}
& \varepsilon_{c}(q)=\bar{\varepsilon}_{c}(k(q)) \text { and } \varepsilon_{s}\left(q^{\prime}\right)=\bar{\varepsilon}_{s}\left(\Lambda\left(q^{\prime}\right)\right), \text { where } \\
& \bar{\varepsilon}_{c}(k)=\int_{Q}^{k} d k^{\prime} 2 t \eta_{c}\left(k^{\prime}\right) \\
& \bar{\varepsilon}_{s}(\Lambda)=\int_{\infty}^{\Lambda} d \Lambda^{\prime} 2 t \eta_{s}\left(\Lambda^{\prime}\right)
\end{aligned}
$$

The distributions $2 t \eta_{c}(k)$ and $2 t \eta_{s}(\Lambda)$ appearing here are solutions of the coupled integral equations,

$$
2 t \eta_{c}(k)=2 t \sin k+\frac{\cos k}{\pi u} \int_{-\infty}^{\infty} d \Lambda \frac{2 t \eta_{s}(\Lambda)}{1+\left(\frac{\sin k-\Lambda}{u}\right)^{2}}
$$

and

$$
\begin{aligned}
2 t \eta_{s}(\Lambda)= & \frac{1}{\pi u} \int_{-Q}^{Q} d k \frac{2 t \eta_{c}(k)}{1+\left(\frac{\Lambda-\sin k}{u}\right)^{2}} \\
& -\frac{1}{2 \pi u} \int_{-\infty}^{\infty} d \Lambda^{\prime} \frac{2 t \eta_{s}\left(\Lambda^{\prime}\right)}{1+\left(\frac{\Lambda-\Lambda^{\prime}}{2 u}\right)^{2}}
\end{aligned}
$$

The rapidity distribution functions $k(q)$ and $\Lambda\left(q^{\prime}\right)$ for the $c$ and $s$ impurity occupancies $q \in\left[-2 k_{F}, 2 k_{F}\right]$ and $q^{\prime} \in$ $\left[-k_{F}, k_{F}\right]$, respectively, in the arguments of the auxiliary dispersions $\bar{\varepsilon}_{c}$ and $\bar{\varepsilon}_{s}$ in Eq. (A4) are defined in terms of their inverse functions $q=q(k)$ where $k \in[-Q, Q]$ and $q^{\prime}=q^{\prime}(\Lambda)$ where $\Lambda \in[-\infty, \infty]$, respectively. The latter are defined by the equations,

$$
\begin{aligned}
q(k)= & k+\frac{1}{\pi} \int_{-\infty}^{\infty} d \Lambda 2 \pi \sigma(\Lambda) \arctan \left(\frac{\sin k-\Lambda}{u}\right) \\
& \text { for } k \in[-Q, Q], \\
q^{\prime}(\Lambda)= & \frac{1}{\pi} \int_{-Q}^{Q} d k 2 \pi \rho(k) \arctan \left(\frac{\Lambda-\sin k}{u}\right) \\
& -\frac{1}{\pi} \int_{-\infty}^{\infty} d \Lambda^{\prime} 2 \pi \sigma\left(\Lambda^{\prime}\right) \arctan \left(\frac{\Lambda-\Lambda^{\prime}}{2 u}\right) \\
& \text { for } \in[-\infty, \infty] .
\end{aligned}
$$

The parameter $Q$ in Eqs. (A4), (A6), and (A7) is defined by the relations,

$$
Q=k\left(2 k_{F}\right) \text { and } q(Q)=2 k_{F} .
$$

Furthermore, the distributions $2 \pi \rho(k)$ and $2 \pi \sigma(\Lambda)$ in Eq. (A7) are the solutions of the coupled integral equations:

$$
2 \pi \rho(k)=1+\frac{\cos k}{\pi u} \int_{-\infty}^{\infty} d \Lambda \frac{2 \pi \sigma(\Lambda)}{1+\left(\frac{\sin k-\Lambda}{u}\right)^{2}}
$$

and

$$
\begin{aligned}
2 \pi \sigma(\Lambda)= & \frac{1}{\pi u} \int_{-Q}^{Q} d k \frac{2 \pi \rho(k)}{1+\left(\frac{\Lambda-\sin k}{u}\right)^{2}} \\
& -\frac{1}{2 \pi u} \int_{-\infty}^{\infty} d \Lambda^{\prime} \frac{2 \pi \sigma\left(\Lambda^{\prime}\right)}{1+\left(\frac{\Lambda-\Lambda^{\prime}}{2 u}\right)^{2}} .
\end{aligned}
$$

In the $u \rightarrow 0$ and $u \gg 1$ limits, the solution and the use of Eqs. (A4)-(A10) leads to the following analytical expressions for the dispersions $\varepsilon_{c}(q)$ and $\varepsilon_{s}\left(q^{\prime}\right)$,

$$
\begin{aligned}
& \varepsilon_{c}(q)=-4 t\left(\cos \left(\frac{q}{2}\right)-\cos k_{F}\right) \text { for } q \in\left[-2 k_{F}, 2 k_{F}\right], \\
& \varepsilon_{s}\left(q^{\prime}\right)=-2 t\left(\cos q^{\prime}-\cos k_{F}\right) \text { for } q^{\prime} \in\left[-k_{F}, k_{F}\right],
\end{aligned}
$$

and

$$
\begin{aligned}
\varepsilon_{c}(q)= & -2 t\left(\cos q-\cos 2 k_{F}\right)-\frac{2 t n_{e} \ln 2}{u}\left(\sin ^{2} q-\sin ^{2} 2 k_{F}\right) \\
& \text { for } q \in\left[-2 k_{F}, 2 k_{F}\right], \\
\varepsilon_{s}\left(q^{\prime}\right)= & -\frac{\pi n_{e} t}{2 u}\left(1-\frac{\sin 2 \pi n_{e}}{2 \pi n_{e}}\right) \cos \left(\frac{q}{n_{e}}\right) \\
& \text { for } q \in\left[-k_{F}, k_{F}\right],
\end{aligned}
$$

respectively.

The bare phase shifts $2 \pi \Phi_{c, s}\left( \pm 2 k_{F}, q^{\prime}\right)$ and $2 \pi \Phi_{c, c}\left( \pm 2 k_{F}, q\right)$ in the expressions of the phase shifts $2 \pi \tilde{\Phi}_{c, s}\left( \pm 2 k_{F}, q^{\prime}\right)$ and $2 \pi \tilde{\Phi}_{c, c}\left( \pm 2 k_{F}, q\right)$ provided in Eqs. (3) and (14), respectively, are given by

$$
\begin{aligned}
& 2 \pi \Phi_{c, c}\left( \pm 2 k_{F}, q\right)=2 \pi \bar{\Phi}_{c, c}\left( \pm \frac{\sin Q}{u}, \frac{\sin k(q)}{u}\right), \\
& 2 \pi \Phi_{c, s}\left( \pm 2 k_{F}, q^{\prime}\right)=2 \pi \bar{\Phi}_{c, s}\left( \pm \frac{\sin Q}{u}, \frac{\Lambda\left(q^{\prime}\right)}{u}\right),
\end{aligned}
$$

where the quantities $2 \pi \bar{\Phi}_{c, c}\left( \pm r_{Q}, r^{\prime}\right)$ and $2 \pi \bar{\Phi}_{c, s}\left( \pm r_{Q}, r^{\prime}\right)$ where $r_{Q}=\frac{\sin Q}{u}$ are particular cases of the rapidity dependent auxiliary phase shifts $2 \pi \bar{\Phi}_{c, c}\left(r, r^{\prime}\right)$ and $2 \pi \bar{\Phi}_{c, s}\left(r, r^{\prime}\right)$. Those are the solution of the following integral equations:

$$
\begin{aligned}
2 \pi \bar{\Phi}_{c, c}\left(r, r^{\prime}\right)= & D_{0}\left(r-r^{\prime}\right)+\int_{-\frac{\sin Q}{u}}^{\frac{\sin Q}{u}} d r^{\prime \prime} \\
& \times D\left(r-r^{\prime \prime}\right) 2 \pi \bar{\Phi}_{c, c}\left(r^{\prime \prime}, r^{\prime}\right)
\end{aligned}
$$

and

$$
\begin{aligned}
2 \pi \bar{\Phi}_{c, s}\left(r, r^{\prime}\right)= & -\arctan \left[\sinh \left(\frac{\pi}{2}\left(r-r^{\prime}\right)\right)\right]+\int_{-\frac{\sin Q}{u}}^{\frac{\sin Q}{u}} d r^{\prime \prime} \\
& \times D\left(r-r^{\prime \prime}\right) 2 \pi \bar{\Phi}_{c, s 1}\left(r^{\prime \prime}, r^{\prime}\right),
\end{aligned}
$$

respectively, where

$$
\begin{gathered}
D_{0}(r)=-2 \int_{0}^{\infty} d \omega \frac{\sin (\omega r)}{\omega\left(1+e^{2 \omega}\right)} \\
=\frac{i}{2 \pi} \ln \frac{\Gamma\left(\frac{1}{2}+i \frac{r}{4}\right) \Gamma\left(1-i \frac{r}{4}\right)}{\Gamma\left(\frac{1}{2}-i \frac{r}{4}\right) \Gamma\left(1+i \frac{r}{4}\right)}, \\
D(r)=-\frac{1}{2 \pi} \frac{d D_{0}(r)}{d r}=\frac{1}{\pi} \int_{0}^{\infty} d \omega \frac{\cos (\omega r)}{1+e^{2 \omega}} \\
=\frac{i}{2 \pi} \frac{d}{d r} \ln \frac{\Gamma\left(\frac{1}{2}+i \frac{r}{4}\right) \Gamma\left(1-i \frac{r}{4}\right)}{\Gamma\left(\frac{1}{2}-i \frac{r}{4}\right) \Gamma\left(1+i \frac{r}{4}\right)},
\end{gathered}
$$

and $\Gamma(z)$ is the usual $\Gamma$ function. 
In the $u \rightarrow 0$ and $u \gg 1$ limits the solution and the use of Eqs. (A11)-(A15) leads to the following analytical expressions for the bare phase shifts $2 \pi \Phi_{c, c}\left( \pm 2 k_{F}, q\right)$ and $2 \pi \Phi_{c, s}\left( \pm 2 k_{F}, q^{\prime}\right)$ :

$$
\begin{aligned}
& \left.2 \pi \Phi_{c, c}\left( \pm 2 k_{F}, q\right)=\mp \frac{\pi}{\sqrt{2}} \text { for } q \in\right]-2 k_{F}, 2 k_{F}[, \\
& \left.2 \pi \Phi_{c, s}\left(\iota 2 k_{F}, q^{\prime}\right)=\mp \frac{\pi}{\sqrt{2}} \text { for } q^{\prime} \in\right]-k_{F}, k_{F}[
\end{aligned}
$$

and

$$
\begin{aligned}
2 \pi \Phi_{c, c}\left( \pm 2 k_{F}, q\right)= & \frac{\ln 2}{u}\left(\mp \sin 2 k_{F}+\sin q\right) \\
& \text { for } q \in]-2 k_{F}, 2 k_{F}[, \\
2 \pi \Phi_{c, s}\left( \pm 2 k_{F}, q^{\prime}\right)= & \frac{q^{\prime}}{n_{e}} \mp \frac{\pi}{2 u} \sin 2 k_{F} \cos \left(\frac{\pi q^{\prime}}{2 k_{F}}\right) \\
& \left.+q^{\prime} \frac{\ln 2}{u} \frac{\sin 2 k_{F}}{2 k_{F}} \text { for } q^{\prime} \in\right]-k_{F}, k_{F}[,
\end{aligned}
$$

respectively

The dependence on the electronic density $\left.n_{e} \in\right] 0,1[$ and interaction $u=U / 4 t$ of the bare charge parameter $\xi_{c}$ is defined by the following relation and equation:

$$
\begin{aligned}
& \xi_{c}= \xi_{c}\left(\frac{\sin Q}{u}\right), \text { where } \xi_{c}(r) \text { is the solution of } \\
& \text { the integral equation, } \\
& \xi_{c}(r)=1+\int_{-\frac{\sin Q}{u}}^{\frac{\sin Q}{u}} d r^{\prime} D\left(r-r^{\prime}\right) \xi_{c}\left(r^{\prime}\right),
\end{aligned}
$$

where $D(r)$ is given in Eq. (A15). Its limiting behaviors are

$$
\begin{aligned}
\xi_{c} & =\sqrt{2}\left(1-\frac{u}{2 \pi \sin Q}\right) \text { for } u \ll 1 \\
& =1+\frac{\ln 2}{\pi u} \sin 2 k_{F} \text { for } u \gg 1,
\end{aligned}
$$

where $\lim _{u \rightarrow 0} Q=k_{F}$ for $\left.n_{e} \in\right] 0,1[$.

Finally, the extended domain of relative fluctuation $\Delta a / \tilde{a}$, Eq. (11), is briefly discussed. For the physical processes of interest for the problem studied in this paper, $\xi_{c}$ in Eq. (A16) varies in the domain $\left.\xi_{c} \in\right] 1, \sqrt{2}$ [. The relative fluctuation $\Delta a / \tilde{a}$ in Eq. (11) where $\Delta a=a-\tilde{a}$ applies though to all finite negative values ] $-\infty, 0[$ of $a / L$ and $\tilde{a} / L$. This refers to an extended domain $\left.\xi_{c} \in\right] 1,2[$.

Its new subinterval $\xi_{c} \in[\sqrt{2}, 2$ [ corresponds to electronic potentials of the same general form, $V_{e}(0)=U / 2$ and $V_{e}(r)=$ $U F_{e}(r) / r$ for $r>0$, as those in Eq. (1) but for which $U \in$ ] $-\infty, 0\left[\right.$. In the general case there are $\xi_{c} \rightarrow \tilde{\xi}_{c}$ transformations within which the function $F_{e}(r)$ is smoothly turned on from $F_{e}(r)=0$ at $\tilde{\xi}_{c}=\xi_{c}$ to (i) positive $F_{e}(r)>0$ and (ii) negative $F_{e}(r)<0$ values, respectively. Considering all such processes and $U \in]-\infty, \infty$ [ values leads to charge parameters that vary in the intervals $\left.\xi_{c} \in\right] 1,2\left[\right.$ and $\left.\tilde{\xi}_{c} \in\right] 1 / 2,2[$, respectively, for which the relations given in Eqs. (12) and (13) remain valid. The scattering length symmetry relations, $a\left(\xi_{c}\right)=a\left(1 / \xi_{c}\right)$ and $\tilde{a}\left(\tilde{\xi}_{c}\right)=\tilde{a}\left(1 / \tilde{\xi}_{c}\right)$, then confirm that both $a / L \in]-\infty, 0[$ and $\tilde{a} / L \in]-\infty, 0[$ in $\Delta a / \tilde{a}$.

Within the new subinterval $\tilde{\xi}_{c} \in[\sqrt{2}, 2[$ the SDS exponent $\alpha$ varies in the physically irrelevant range $\alpha \in[0,1 / 8]$. $\alpha=1 / 8$ refers here to $\tilde{\xi}_{c} \rightarrow 2$. Only $\xi_{c} \rightarrow \tilde{\xi}_{c}$ transformations for which both $\left.\xi_{c} \in\right] 1, \sqrt{2}\left[\right.$ and $F_{e}(r)>0$ refer to processes contributing to the physical problem studied in this paper.

\section{APPENDIX B: RELATION TO THE TLL REGIME AND CROSSOVER TO IT}

Both the MQIM-LO and the MQIM-HO also apply to the low-energy TLL regime whose spectral-function exponents near the $c, c^{\prime}, s$ branch lines are different from those given in Eq. (A3). In the high-energy regime whose spectral function expression, Eq. (2), was used in this paper to predict the $(k, \omega)$-plane location of the high-energy $\mathrm{Bi} / \mathrm{InSb}(001)$ ARPES peaks, the velocity of the $c$ or $s$ impurity is different from the velocity at the $c$ or $s$ band Fermi points, respectively.

In contrast, in the TLL regime the (i) $c$ or (ii) $s$ impurity is created in its band at a momentum in one of the intervals (i) $q \in\left[-2 k_{F},-2 k_{F}+k_{F c}^{0}\right]$ and $q \in\left[2 k_{F}-k_{F c}^{0}, 2 k_{F}\right]$ or (ii) $q^{\prime} \in\left[-k_{F},-k_{F}+k_{F s}^{0}\right]$ and $q^{\prime} \in\left[k_{F}-k_{F s}^{0}, k_{F}\right]$. (Here both $k_{F c}^{0} / 2 k_{F} \ll 1$ and $\left.k_{F s}^{0} / k_{F} \ll 1\right)$. The group velocity of that impurity thus becomes that of the low-energy particlehole excitations near the corresponding Fermi point (i) $-2 k_{F}$ and $2 k_{F}$ or (ii) $-k_{F}$ and $k_{F}$, respectively. Hence they loses their identity, as they cannot be distinguished from the $c$ or $s$ holes (usual holons and spinons) in such excitations.

The exponents in Eq. (A3) can be rewritten as $\tilde{\zeta}_{\gamma}=-1+$ $\sum_{\iota= \pm 1}\left(2 \tilde{\Phi}_{c}^{\iota}+2 \tilde{\Delta}_{s}^{\iota}\right)$. Here, $2 \tilde{\Phi}_{c}^{\iota}=\left(-\iota / 2 \tilde{\xi}_{c}-\tilde{\Phi}_{c, s}\left(\iota 2 k_{F}, q^{\prime}\right)\right)^{2}$ and $2 \tilde{\Delta}_{s}^{\iota}=0$ for the one-electron removal $s$ branch line and $2 \tilde{\Phi}_{c}^{\iota}=\left(\tilde{\xi}_{c} / 4-\tilde{\Phi}_{c, c}\left(\iota 2 k_{F}, q\right)\right)^{2}$ and $2 \tilde{\Delta}_{s}^{\iota}=\frac{1}{8}(1+\iota)^{2}$ for both the one-electron removal $c$ and $c^{\prime}$ branch lines, which correspond to different intervals of the $c$ band momentum $q$ in $\tilde{\Phi}_{c, c}\left(\iota 2 k_{F}, q\right)$.

It turns out that in the TLL regime the expressions for the $\gamma=c, c^{\prime}, s$ exponents in the above equation lose one of the four $2 \tilde{\Delta}_{\gamma}^{\iota}$ s. It is the $2 \tilde{\Delta}_{\gamma}^{\iota}$ whose sign of $\iota= \pm 1$ is that of the Fermi point whose velocity is the same as the $\gamma=c$ or $\gamma=s$ impurity velocity. The expressions of the exponents, Eq. (A3), in the high-energy spectral function expressions (2) used in the theoretical study of the $\mathrm{Bi} / \mathrm{InSb}(001)$ high-energy ARPES peaks are thus different from those of the TLL regime.

In the case of a large finite system, there is a cross-over regime between the high-energy regime and the low-energy TLL regime within which the above quantity $2 \tilde{\Phi}_{c}^{\iota}$ or $2 \tilde{\Delta}_{s}^{\iota}$ is gradually removed as the energy decreases. This cross-over regime refers to $(k, \omega)$-plane regions whose momentum and energy widths are very small or vanish in the thermodynamic limit. It is an interesting theoretical problem, but the details of its physics have no impact on the specific problems discussed in this paper.

\section{APPENDIX C: ELECTRON AND $c$ PARTICLE REPRESENTATIONS}

In the bare limit, $\tilde{\xi}_{c}=\xi_{c}$, the $c$ particles are directly related to rotated electrons for which double occupancy is a good quantum number for $u>0$. Their operators

$$
\tilde{c}_{j, \sigma}^{\dagger}=\hat{U}^{\dagger} c_{j, \sigma}^{\dagger} \hat{U}, \quad \tilde{c}_{j, \sigma}=\hat{U}^{\dagger} c_{j, \sigma} \hat{U}, \quad \tilde{n}_{j, \sigma}=\tilde{c}_{j, \sigma}^{\dagger} \tilde{c}_{j, \sigma},
$$


and $\tilde{n}_{j}=\sum_{\sigma} \tilde{n}_{j, \sigma}$ are generated from those of the electrons by the unitary operator $\hat{U}=e^{\hat{s}}$. It is uniquely defined in Ref. [40] in terms of the $4^{L} \times 4^{L}$ matrix elements between all the model energy eigenstates.

The present quantum problem is defined in a subspace without rotated-electron double occupancy. Hence $\hat{U}=e^{\hat{s}}$ merely removes the corresponding electron double occupancy from all sites around that of index $j$ at which $\tilde{c}_{j, \sigma}^{\dagger}$ or $\tilde{c}_{j, \sigma}$ acts. In that subspace, the electron double occupancy reads $D=N_{e} \frac{n_{e}}{4} f\left(n_{e}, u\right)$ where $f\left(n_{e}, u\right)=\frac{\ln 2}{u^{2}}\left(1-\frac{\sin \left(2 \pi n_{e}\right)}{2 \pi n_{e}}\right)$ for $u \gg 1$ and $\lim _{u \rightarrow 0} f\left(n_{e}, u\right)=1, \tilde{c}_{j, \uparrow}^{\dagger}=\left(\frac{1}{2}-\tilde{S}_{j, s}^{z}\right) f_{j, c}^{\dagger}$, and $\tilde{c}_{j, \downarrow}^{\dagger}=\tilde{S}_{j, s}^{+} f_{j, c}^{\dagger}$. Here, $f_{j, c}^{\dagger}$ is the $c$ particle creation operator and $\tilde{S}_{j, s}^{z}$ and $\tilde{S}_{j, s}^{+}$are usual spin operators written in terms of rotated-electron operators.

The same rotated-electron basis and corresponding $c$ particle representation can be used for the $\tilde{\xi}_{c}<\xi_{c}$ renormalized Hamiltonian, Eq. (1). The difference relative to the bare limit is that the states generated from the fractionalized particles configurations are not in general energy eigenstates but in the relevant subspaces for which the $c$ impurity has a large lifetime, they have overlap with single energy eigenstates.

The operator $\hat{U}=e^{\hat{S}}$ preserves the distance $r=j-j^{\prime}$ between electrons, being the same for rotated electrons. The rotated-electron anticommutation relations that follow from unitarity imply that the $c$ particle operators obey a fermionic algebra, $\left\{f_{j, c}^{\dagger}, f_{j^{\prime}, c}\right\}=\delta_{j, j^{\prime}}$ and $\left\{f_{j, c}^{\dagger}, f_{j^{\prime}, c}^{\dagger}\right\}=\left\{f_{j, c}, f_{j^{\prime}, c}\right\}=0$. In the rotated-electron basis the Hamiltonian, Eq. (1), has an infinite number of terms given by the Baker-CampbellHausdorff formula,

$$
\hat{H}=t \tilde{T}+\tilde{V}+[\tilde{H}, \tilde{S}]+\frac{1}{2}[[\tilde{H}, \tilde{S}], \tilde{S}]+\ldots
$$

Here, $\tilde{S}=\hat{U}^{\dagger} \hat{S} \hat{U}=\hat{S}, \tilde{H}=t \tilde{T}+\tilde{V}$ has the same expression in terms of rotated-electron operators as $\hat{H}$ in terms of electron operators, and all higher terms have a kinetic nature. Indeed, the expression of $\tilde{S}$ only involves the three kinetic operators $\tilde{T}_{0}=\sum_{j=1}^{L} \sum_{\iota= \pm 1}\left(\tilde{T}_{0, j, \iota}+\tilde{T}_{0, j, \iota}^{\dagger}\right), \tilde{T}_{+1}=\sum_{j=1}^{L} \sum_{\iota= \pm 1} \tilde{T}_{+1, j, \iota}$, and $\tilde{T}_{-1}=\tilde{T}_{+1}^{\dagger}$ in $\tilde{T}=\sum_{d} \tilde{T}_{d}$ where $d=0, \pm 1$ gives the change in the number of rotated-electron doubly occupied sites, and

$$
\begin{aligned}
\tilde{T}_{0, j, \iota}= & -\sum_{\sigma}\left\{\tilde{n}_{j,-\sigma} \tilde{c}_{j, \sigma}^{\dagger} \tilde{c}_{j+\iota, \sigma} \tilde{n}_{j+\iota,-\sigma}\right. \\
& \left.+\left(1-\tilde{n}_{j,-\sigma}\right) \tilde{c}_{j, \sigma}^{\dagger} \tilde{c}_{j+l, \sigma}\left(1-\tilde{n}_{j+l,-\sigma}\right)\right\} \\
\hat{T}_{+1, j, \iota}= & -\sum_{\sigma}\left\{\tilde{n}_{j,-\sigma} \tilde{c}_{j, \sigma}^{\dagger} \tilde{c}_{j+l, \sigma}\left(1-\tilde{n}_{j+l,-\sigma}\right)\right. \\
& \left.+\tilde{n}_{j+\iota,-\sigma} \tilde{c}_{j+l, \sigma}^{\dagger} \tilde{c}_{j, \sigma}\left(1-\tilde{n}_{j,-\sigma}\right)\right\}
\end{aligned}
$$

Consistent with the finite-range electron interactions having their strongest effects in the charge-charge interaction channel, $\tilde{V}$ in Eq. (C2) can be expressed solely in terms of the charge $c$ particle operators as

$$
\begin{aligned}
\tilde{V}= & \sum_{r=1}^{L / 2-1} V_{e}(r) \sum_{j=1}^{L}\left(1-f_{j, c}^{\dagger} f_{j, c}\right)\left(1-f_{j+r, c}^{\dagger} f_{j+r, c}\right) \\
& +\frac{U}{2} \sum_{j=1}^{L}\left(\frac{1}{2}-f_{j, c}^{\dagger} f_{j, c}\right) .
\end{aligned}
$$

Here, $\quad f_{j, c}^{\dagger}=\left(f_{j, c}\right)^{\dagger}=\tilde{c}_{j, \uparrow}^{\dagger}\left(1-\tilde{n}_{j, \downarrow}\right)+(-1)^{j} \tilde{c}_{j, \uparrow} \tilde{n}_{j, \downarrow} \quad$ for whole Hilbert space where the rotated-electron operators are related to those of the electrons in Eq. (C1).

Importantly, despite the infinite number of terms in the Hamiltonian, Eq. (1), when expressed in terms of the rotatedelectron operators, Eq. (C2), its relevant term for our study is that in Eq. (C4) with $V_{e}(r)$ replaced by rotated-electron potential $V_{r e}(r)$ renormalized by the higher kinetic terms in the expansion, Eq. (C2). The latter are in turn renormalized by $V_{e}(r) .[\tilde{H}, \tilde{S}]$ only involves the $d=0, \pm 1$ operators $\tilde{T}_{d}$ and the four operators $\tilde{J}_{0}^{+}=\left[\tilde{V}, \tilde{T}_{0}\right], \tilde{J}_{0}^{-}=\left(\tilde{J}_{0}^{+}\right)^{\dagger}$, and $\tilde{J}_{ \pm 1}=\left[\tilde{V}, \tilde{T}_{ \pm 1}\right]$,

$$
\begin{aligned}
\tilde{J}_{0}^{+}= & \sum_{r=1}^{L / 2-1} V_{e}(r) \sum_{j=1}^{L} \sum_{\iota= \pm 1}\left(\tilde{T}_{0, j, \iota}-\tilde{T}_{0, j, l}^{\dagger}\right) \\
& \times\left(\tilde{n}_{j+r}+\tilde{n}_{j-r}-\tilde{n}_{j+r+\iota}-\tilde{n}_{j-r+\iota}\right), \\
\tilde{J}_{ \pm 1}= & \pm U \tilde{T}_{ \pm 1} \pm \sum_{r=1}^{L / 2-1} 4 V_{e}(r) \sum_{j=1}^{L} \sum_{l= \pm 1} \tilde{T}_{ \pm 1, j, \iota} \\
& \times\left(\tilde{n}_{j+r}+\tilde{n}_{j-r}+\tilde{n}_{j+r+\iota}+\tilde{n}_{j-r+\iota}\right) .
\end{aligned}
$$

Higher kinetic terms also only involve the operators $\tilde{T}_{0, j, l}$ and $\tilde{T}_{ \pm 1, j, l}$, Eq. (C3), and $\tilde{n}_{j}$ at different relative sites.

The interaction between a $c$ particle at site $j$ and the $c$ impurity at site $j+r$ refers to a Hamiltoninan term of the form $-V_{r e}(r) f_{j, c}^{\dagger} f_{j, c} f_{j+r, c} f_{j+r, c}^{\dagger}$. In terms of $V_{c}(x) \propto-V_{r e}(r)$, it refers to suitably transformed operators $\breve{f}_{j, c}^{\dagger}$ and corresponds to $V_{c}(x) \breve{f}_{j, c}^{\dagger} \breve{j}_{j, c} \breve{f}_{j+x, c} \breve{f}_{j+x, c}^{\dagger}$ [38]. The part of the $V_{r e}(r)$ renormalization by the infinite kinetic energy terms beyond $t \tilde{T}+\tilde{V}$ in Eq. (C2) that contributes to the universal properties is accounted for by the $\xi_{c} \rightarrow \tilde{\xi}_{c}$ transformation. The nonuniversal part is within the nonuniversal inverse reduced mass $\mu^{-1}$ to which $V_{c}(x)$ is also proportional, $V_{c}(x) \propto \frac{1}{2 \mu}$.

The Fourier transform $\mathcal{V}_{r e}(k)$ of $V_{r e}(r)$ controls the $c$ band energy dispersion $\tilde{\varepsilon}_{c}(q)$ and velocity $\tilde{v}_{c}(q)$ renormalization. At $k=0$, it has the universal behavior [38]:

$$
\begin{aligned}
& \mathcal{V}_{r e}(0)=\frac{\pi}{2} \alpha_{c} v_{c}\left(2 k_{F}\right) \text {, where }
\end{aligned}
$$

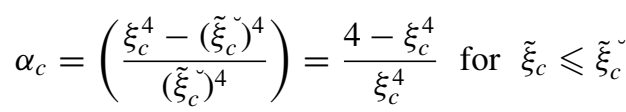

$$
\begin{aligned}
& =\left(\frac{\xi_{c}^{4}-\tilde{\xi}_{c}^{4}}{\tilde{\xi}_{c}^{4}}\right) \text { for } \tilde{\xi}_{c} \geqslant \tilde{\xi}_{c} .
\end{aligned}
$$

Here, $v_{c}\left(2 k_{F}\right)$ is the bare $c$ band velocity $v_{c}(q)$ at $q=2 k_{F}$ defined by Eq. (A2) of Appendix A for $\beta=c, \tilde{\xi}_{c}=\xi_{c}^{2} / \sqrt{2} \in$ ] $1 / \sqrt{2}, 1\left[\right.$, and $\alpha_{c}$ is related to the enhancement parameter $\beta_{c}$ in that equation as $\alpha_{c}=\left(1+\beta_{c}\right)^{2}-1$ and thus determines its value, $\beta_{c}=\sqrt{1+\alpha_{c}}-1$. The $\alpha_{c}$ expression in Eq. (C6) gives $\alpha_{c}=0$ at $U=0$ and $\alpha_{c} \leqslant U /\left(2 \pi t \sin k_{F}\right)$ and $\mathcal{V}_{r e}(0) \leqslant U / 2$ for $u \ll 1$, as required by the properties of the related potential $V_{e}(r)=U F_{e}(r) / r \propto U$ for $r>0$.

On the other hand, the Fourier transform $\mathcal{V}_{c}(k)$ of the potential $V_{c}(x)$ is found in Ref. [38] to read $\mathcal{V}_{c}(0)=-C_{c e} \mathcal{V}_{r e}(0)$ at $k=0$ where the coefficient is given by $C_{c e}=2\left(\xi_{c}^{4}-\right.$ $\left.\tilde{\xi}_{c}^{4}\right) /\left[\tilde{\xi}_{c}^{2} \xi_{c}^{2}\left(4-\xi_{c}^{4}\right)\right]$ for $\tilde{\xi}_{c} \leqslant \tilde{\xi}_{c}$ and $C_{c e}=1$ for $\tilde{\xi}_{c} \geqslant \tilde{\xi}_{c}$. The quantity $\mathcal{V}_{c}(0)$ is related to the phase-shift and matrix-element renormalization. 
[1] C. Blumenstein, J. Schäfer, S. Mietke, S. Meyer, A. Dollinger, M. Lochner, X. Y. Cui, L. Patthey, R. Matzdorf, and R. Claessen, Nat. Phys. 7, 776 (2011).

[2] J. Voit, Rep. Prog. Phys. 58, 977 (1995).

[3] S. Tomonaga, Prog. Theor. Phys. 5, 544 (1950).

[4] J. M. Luttinger, J. Math. Phys. 4, 1154 (1963).

[5] J. M. P. Carmelo, K. Penc, and D. Bozi, Nucl. Phys. B 725, 421 (2005); 737, 351(E) (2006).

[6] A. Imambekov and L. I. Glazman, Science 323, 228 (2009).

[7] A. Imambekov, T. L. Schmidt, and L. I. Glazman, Rev. Mod. Phys. 84, 1253 (2012).

[8] H. J. Schulz, Phys. Rev. Lett. 64, 2831 (1990).

[9] H. Bethe, Z. Phys. 71, 205 (1931).

[10] E. H. Lieb and F. Y. Wu, Phys. Rev. Lett. 20, 1445 (1968).

[11] M. J. Martins and P. B. Ramos, Nucl. Phys. B 522, 413 (1998).

[12] J. M. P. Carmelo and P. D. Sacramento, Phys. Rep. 749, 1 (2018).

[13] R. Claessen, M. Sing, U. Schwingenschlögl, P. Blaha, M. Dressel, and C. S. Jacobsen, Phys. Rev. Lett. 88, 096402 (2002).

[14] B. J. Kim, H. Koh, E. Rothenberg, S.-J. Oh, H. Eisaki, N. Motoyama, S. Uchida, T. Tohyama, S. Maekawa, Z.-H. Shen, and C. Kim, Nat. Phys. 2, 397 (2006).

[15] K. Schönhammer and M. Meden, J. Electron Spectrosc. Relat. Phenom. 62, 225 (1993).

[16] Y. Ma, H. C. Diaz, J. Avila, C. Chen, V. Kalappattil, R. Das, M.-H. Phan, T. Čadež, J. M. P. Carmelo, M. C. Asensio, and M. Batzill, Nat. Commun. 8, 14231 (2017).

[17] Y. Ohtsubo, J.-i. Kishi, K. Hagiwara, P. Le Fèvre, F. Bertran, A. Taleb-Ibrahimi, H. Yamane, S.-i. Ideta, M. Matsunami, K. Tanaka, and S.-i. Kimura, Phys. Rev. Lett. 115, 256404 (2015).

[18] Y. Ohtsubo, J.-I. Kishib, K. Hagiwara, P. Le Fèvre, F. Bertran, A. Taleb-Ibrahimi, and S.-I. Kimura, J. Electron Spectrosc. Relat. Phenom. 220, 37 (2017).

[19] H. A. Bethe, Phys. Rev. 76, 38 (1949).

[20] J. M. Blatt and J. D. Jackson, Phys. Rev. 76, 18 (1949).

[21] C. J. Joachain, Quantum Collision Theory (North-Holland, Amsterdam, 1975).

[22] M. A. Preston and R. K. Bhaduri, Structure of the Nucleus (Addison-Wesley, Reading, Ma, 1975).
[23] V. V. Flambaum, G. F. Gribakin, and C. Harabati, Phys. Rev. A 59, 1998 (1999).

[24] T. Čadež, L. Li, E. V. Castro, and J. M. P. Carmelo, Phys. Rev. B 99, 155109 (2019).

[25] L. D. Landau and E. M. Lifshitz, Quantum Mechanics: NonRelativistic Theory (Pergamon, Oxford, 1965).

[26] M. W. Kermode and W. van Dijk, Phys. Rev. C 42, 1891 (1990).

[27] P. G. Burke, R-Matrix Theory of Atomic Collisions (SpringerVerlag, Heidelberg, 2011), Sec. 1.4.

[28] R. G. Newton, Scattering Theory of Waves and Particles (Springer-Verlag, Berlin, 1982).

[29] W. Zwerger (editor), The BCS-BEC Crossover and the Unitary Fermi Gas, Lecture Notes in Physics Vol. 836 (Springer-Verlag, Heidelberg, 2012).

[30] M. Horikoshi, M. Koashi, H. Tajima, Y. Ohashi, and M. Kuwata-Gonokami, Phys. Rev. X 7, 041004 (2017).

[31] D. J. Dean and M. Hjorth-Jenson, Rev. Mod. Phys. 75, 607 (2003).

[32] G. F. Gribakin and V. V. Flambaum, Phys. Rev. A 48, 546 (1993).

[33] D. Orgad, S. A. Kivelson, E. W. Carlson, V. J. Emery, X. J. Zhou, and Z. X. Shen, Phys. Rev. Lett. 86, 4362 (2001).

[34] J. Rollbühler and H. Grabert, Phys. Rev. Lett. 87, 126804 (2001).

[35] E. G. Mishchenko, A. V. Andreev, and L. I. Glazman, Phys. Rev. Lett. 87, 246801 (2001).

[36] L. Bartoscha and P. Kopietz, Eur. Phys. J. B 28, 29 (2002)

[37] J. Kishi, Y. Ohtsubo, T. Nakamura, K. Yaji, A. Harasawa, F. Komori, S. Shin, J. E. Rault, P. Le Fèvre, F. Bertran, A. TalebIbrahimi, M. Nurmamat, H. Yamane, S. Ideta, K. Tanaka, and S. Kimura, Phys. Rev. Mater. 1, 064602 (2017).

[38] J. M. P. Carmelo, T. Čadež, D. K. Campbell, M. Sing, and R. Claessen (unpublished).

[39] M. Hohenadler, S. Wessel, M. Daghofer, and F. F. Assaad, Phys. Rev. B 85, 195115 (2012).

[40] J. M. P. Carmelo and T. Čadež, Nucl. Phys. B 914, 461 (2017).

[41] Y. Castin and F. Werner, Lect. Notes Phys. 836, 127 (2012). 